\title{
Study of the Initial Value Problems Appearing in a Factorization Method of Second Order Elliptic Boundary Value Problems
}

\author{
J. Henry ${ }^{\mathrm{a}}$, A.M. Ramos ${ }^{\mathrm{b}, 1 *}$ \\ ${ }^{a}$ Institut de Mathématiques - MAB, INRIA Futurs - Bordeaux, Université Bordeaux 1, \\ 351, cours de la libération, 33405 Talence cedex, France. \\ b Departamento de Matemática Aplicada, Universidad Complutense de Madrid, \\ Plaza de Ciencias 3, 28040, Madrid, Spain.
}

\begin{abstract}
In [8] we presented a method to factorize a second order boundary value problem into a system of uncoupled first order initial value problems, together with a nonlinear Riccati type equation for functional operators. A weak sense was given to that system but we did not perform a direct study of those equations. This factorization utilizes either the Neumann to Dirichlet (NtD) operator or the Dirichlet to Neumann (DtN) operator, which satisfiy a Riccati equation. Here we consider the framework of Hilbert-Schmidt operators, which provides tools for a direct study of this Riccati type equation. Once we have solved the system of Cauchy problems, we show that its solution solves the original second order boundary value problem. Finally, we indicate how this techniques can be used to find suitable transparent conditions.
\end{abstract}

keywords: Factorization, boundary value problem, Hilbert-Schmidt operator, Riccati equation; invariant embedding; Neumann-to-Dirichlet (NtD) operator; Dirichlet-to-Neumann (DtN) operator, transparent conditions.

\section{Introduction}

In [1] Angel and Bellman proposed a method based on spatial invariant embedding to transform a second order elliptic boundary value problem in a rectangle in a system of first

\footnotetext{
${ }^{*}$ Corresponding author. Tel.: +34-91-3944480; fax: +34-91-3944613.

E-mail addresses: Jacques.Henry@inria.fr(J. Henry), Angel_Ramos@mat.ucm.es (A.M. Ramos).

${ }^{1}$ Supported by the Spanish 'Plan Nacional de I+D+I (2000-2003) del MCYT', through the AGL200306862-C02-02 project.
} 
order decoupled initial value problems which can be solved by a two sweep process. In [8] Henry and Ramos gave a complete justification for this transform for the Poisson equation in a $n$-dimensional cylindrical domain. The invariant embedding was performed using the coordinate along the axis of the cylinder. The Neumann-to-Dirichlet (NtD) operator on a section of the cylinder was shown to satisfy a Riccati equation. The relationship of this method with the similar one to derive the optimal feedback for optimal control problems of parabolic equations was described. The study of the well-posedness of the Riccati equation was similar to the one used by Lions [9] and based on a Galerkin method. Furthermore the relationship between this factorization method and the block $L U$ factorization for a discretized version of the problem was established.

In this paper we consider a similar problem, allowing for a diffusivity coefficient depending on the coordinate along the axis of the cylinder. Following the paper by Temam [13] we study directly the Riccati equation, that is without reference to the boundary value problem (as it was done in [8]), in a Hilbert-Schmidt framework. We cannot apply the results of [13] as here the operator appearing in the quadratic term is unbounded (the same difficulty was encountered in [8]). The Hilbert-Schmidt framework appears to be efficient for the studied problem, using a fixed basis of eigenfunctions of the Laplacian restricted to the section. Although it defines the solution in a weaker sense than the one that could be hoped, this regularity is easily recovered. The solution of the boundary value problem is then obtained in factorized form, that is by solving two uncoupled Cauchy problems. This technique can be used to find suitable transparent boundary condition in a subdomain.

In section 2 we recall the formal derivation of the factorization. We recall some HilbertSchmidt properties from [13] in section 3. The study of the Riccati equation is done in section 4 . The factorized form of the boundary value problem is obtained in section 5 . The initial definition of $P$ as a NtD operator given in [8] is recovered and its use to build transparent boundary conditions is presented.

\section{The Factorization Method for Boundary Value Prob- lems}

Let $\Omega$ be the cylinder $\Omega=] 0, a\left[\times \mathcal{O}\right.$ in $\mathbb{R}^{d}$, with $\mathcal{O}$ a bounded open set in $\mathbb{R}^{d-1}$ and $\Gamma_{s}=\{s\} \times \mathcal{O}$. The lateral boundary of the cylinder is denoted by $\left.\Sigma=\right] 0, a[\times \partial \mathcal{O}$ and a general point $\left(x_{1}, x_{2}, \cdots, x_{d}\right) \in \Omega$ is also denoted by $(x, y)$, where $x=x_{1}$ and $y$ denotes the independent variables $\left(x_{2}, \ldots, x_{d}\right)$. We use the Hilbert space $H_{00}^{1 / 2}(\mathcal{O})=\left[H_{0}^{1}(\mathcal{O}), L^{2}(\mathcal{O})\right]_{1 / 2}$ defined in [10]. Let $f \in L^{2}(\Omega), y_{0} \in H_{00}^{1 / 2}(\mathcal{O}), y_{a} \in H_{00}^{1 / 2}(\mathcal{O})^{\prime}$ (respectively $y_{a} \in H_{00}^{1 / 2}(\mathcal{O})$ ) and $\alpha \in L^{\infty}(0, a)$ (the study of the particular case with $\alpha \in \mathbb{R}$ constant can be seen in [4]) with

$$
0<\bar{\alpha}=\inf _{x \in[0, a]} \alpha(x) \leq \underline{\alpha}=\sup _{x \in[0, a]} \alpha(x) .
$$

Let us consider the problem 


$$
\begin{cases}-\nabla \cdot(\alpha(x) \nabla u)=f & \text { in } \Omega, \\ u=0 & \text { on } \Sigma, \\ u=u_{0} & \text { on } \Gamma_{0}, \\ \alpha \frac{\partial u}{\partial x}=u_{a} & \text { on } \Gamma_{a} \quad\left(\text { respectively } u=u_{a} \text { on } \Gamma_{a}\right) .\end{cases}
$$

Let us embed this problem in a family of similar problems defined in sub-cylinders $\Omega_{s}=$ ] $0, s\left[\times \mathcal{O}\right.$ for $0<s<a$, with the boundary data $\alpha \frac{\partial u}{\partial x}=h$ on $\Gamma_{s}$. This family depends on $s$ and $h$. By linearity, we have the existence of an operator $P$ and a function $r$ satisfying

$$
\left.u\right|_{\Gamma_{s}}=P(s) h+r(s) .
$$

For a solution of (1) this identity is verified for any $x$ :

$$
u(x)=P(x) \alpha(x) \frac{\partial u}{\partial x}(x)+r(x) .
$$

From now on we shall consider $u$ as a function of $x$ with values in a function space defined on $\mathcal{O}$, so we use the notation $\frac{\mathrm{d}}{\mathrm{d} x}$ for the $x$-derivation. By formal derivation (to be justified later), we have

$$
\begin{aligned}
\frac{\mathrm{d} u}{\mathrm{~d} x} & =\frac{\mathrm{d} P}{\mathrm{~d} x} \alpha(x) \frac{\mathrm{d} u}{\mathrm{~d} x}+P \frac{\mathrm{d}}{\mathrm{d} x}\left(\alpha(x) \frac{\mathrm{d} u}{\mathrm{~d} x}\right)+\frac{\mathrm{d} r}{\mathrm{~d} x} \\
& =\frac{\mathrm{d} P}{\mathrm{~d} x} \alpha(x) \frac{\mathrm{d} u}{\mathrm{~d} x}-P \alpha \Delta_{y} P \alpha \frac{\mathrm{d} u}{\mathrm{~d} x}-P \alpha \Delta_{y} r-P f+\frac{\mathrm{d} r}{\mathrm{~d} x}
\end{aligned}
$$

Therefore

$$
\left(\frac{\mathrm{d} P}{\mathrm{~d} x}-\alpha P \Delta_{y} P-\alpha^{-1} I\right) \alpha \frac{\mathrm{d} u}{\mathrm{~d} x}+\left(\frac{\mathrm{d} r}{\mathrm{~d} x}-\alpha P \Delta_{y} r-P f\right)=0 .
$$

Then, we have

$$
\left\{\begin{array}{l}
\frac{\mathrm{d} P}{\mathrm{~d} x}-\alpha P \Delta_{y} P-\alpha^{-1} I=0, \quad P(0)=0, \\
\frac{\mathrm{d} r}{\mathrm{~d} x}-\alpha P \Delta_{y} r=P f, \quad r(0)=u_{0}, \\
-P \alpha \frac{\mathrm{d} u}{\mathrm{~d} x}+u=r, \quad u(a)=P(a) u_{a}+r(a)\left(\text { respectively } u(a)=u_{a}\right) .
\end{array}\right.
$$

We point out that we have factorized the equation appearing in (1) as

$$
\left(\frac{\mathrm{d}}{\mathrm{d} x}-\alpha P \Delta_{y}\right)\left(I-\alpha P \frac{\mathrm{d}}{\mathrm{d} x}\right) u=P f
$$


which is equivalent, by using that $\frac{\mathrm{d}}{\mathrm{d} x} P=\frac{\mathrm{d} P}{\mathrm{~d} x}+P \frac{\mathrm{d}}{\mathrm{d} x}$ and the Riccati equation to

$$
\left(\frac{\mathrm{d}}{\mathrm{d} x}+P^{-1} \alpha^{-1}\right)\left(P^{-1}-\alpha \frac{\mathrm{d}}{\mathrm{d} x}\right) u=f,
$$

or, in self-adjoint form,

$$
\left(\frac{\mathrm{d}}{\mathrm{d} x} \alpha^{1 / 2}+P^{-1} \alpha^{-1 / 2}\right)\left(\alpha^{-1 / 2} P^{-1}-\alpha^{1 / 2} \frac{\mathrm{d}}{\mathrm{d} x}\right) u=f .
$$

Remark that $P^{-1}$ is not defined at 0 .

Remark 1 Operator $P$ is a Neumann to Dirichlet operator (NtD). We could have also used a Dirichlet to Neumann operator, which gives the system

$$
\left\{\begin{array}{l}
-\frac{\mathrm{d} Q}{\mathrm{~d} x}+\alpha^{-1} Q^{2}-\alpha \Delta_{y}=0, \quad Q(a)=0 \\
\frac{\mathrm{d} w}{\mathrm{~d} x}-\alpha^{-1} Q w=f, \quad w(a)=u_{a} \\
\alpha \frac{\mathrm{d} u}{\mathrm{~d} x}+Q u=-w, \quad u(0)=u_{0} .
\end{array}\right.
$$

We point out that we have factorized the equation appearing in (1) as

$$
-\left(\frac{\mathrm{d}}{\mathrm{d} x}-\alpha^{-1} Q\right)\left(\alpha \frac{\mathrm{d}}{\mathrm{d} x}+Q\right) u=f
$$

or, in self-adjoint form,

$$
-\left(\frac{\mathrm{d}}{\mathrm{d} x}-\alpha^{-1} Q\right) \alpha\left(\frac{\mathrm{d}}{\mathrm{d} x}+\alpha^{-1} Q\right) u=f .
$$

In the following sections we carry out a rigorous mathematical study of system (2). In contrast with other studies of this system (see [8]) this study will be done directly, without considering the fact that those equations come from a factorization method. We show existence and uniqueness results for the solution of system (2) and we show that we recover the solution of problem (1).

\section{Hilbert-Schmidt Spaces}

\subsection{Generalities}

In this section we recall the definitions and properties of Hilbert-Schmidt spaces (see [2] and [5]) following the notation given in [13]. We consider two Hilbert spaces $X, Y$ with scalar products $(\cdot, \cdot)_{X},(\cdot, \cdot)_{Y}$ and associated norms $\|\cdot\|_{X},\|\cdot\|_{Y}$. We suppose that $X$ and $Y$ are separable and $\left\{\varphi_{i}\right\}_{i=1}^{\infty}\left\{\psi_{i}\right\}_{i=1}^{\infty}$ are any orthonormal basis of $X$ and $Y$ respectively. 
Definition $2 P \in \mathcal{L}(X, Y)$ (a bounded linear operator from $X$ to $Y$ ) is said to be a Hilbert-Schimdt operator in case

$$
\sum_{i=1}^{\infty}\left\|P\left(\varphi_{i}\right)\right\|_{Y}^{2}<\infty
$$

In this case we denote $P \in X \widehat{\otimes}_{2} Y$ and

$$
\|P\|_{X \widehat{\otimes}_{2} Y}=\left(\sum_{i=1}^{\infty}\left\|P\left(\varphi_{i}\right)\right\|_{Y}^{2}\right)^{1 / 2}
$$

is called the Hilbert-Schmidt norm of $P$.

The proof of the following properties and other results regarding Hilbert-Schmidt spaces can be seen in [5] and [2].

Lemma 3 The Hilbert-Schmidt norm is independent of the orthonormal basis used in its definition and

$$
\|P\|_{X \widehat{\otimes}_{2} Y}=\left(\sum_{i, j=1}^{\infty}\left|\left(P \varphi_{i}, \psi_{j}\right)_{Y}\right|^{2}\right)^{1 / 2} .
$$

Proposition 4 Every Hilbert-Schmidt operator is compact.

Proposition $5 \mathrm{X} \widehat{\otimes}_{2} Y$ is a Hilbert space with the inner product

$$
[P, Q]_{X \widehat{\otimes}_{2} Y}=\sum_{i=1}^{\infty}\left(P \varphi_{i}, Q \varphi_{i}\right)_{Y}
$$

\subsection{A special case}

Following [10], let us suppose that $H, V$ are two real separable Hilbert spaces such that $V \subset H$ and $V$ is dense in $H$ with continuous injection. Then, we can identify $H^{\prime}$ (topological dual of $H$ ) with $H$ and with a dense subset of $V^{\prime}$. Therefore,

$$
V \subset H \subset V^{\prime}
$$

with every space being dense in the following one with continuous injections.

Let us suppose that the injection $V \subset H$ is compact and let $\Lambda: V \rightarrow V^{\prime}$ be the canonical isomorphism between $V$ and $V^{\prime}$, i.e.

$$
(\Lambda u, v)_{V^{\prime} \times V}=(u, v)_{V} \quad \forall u, v \in V .
$$

Then, $\Lambda^{-1}: H \rightarrow V$ is a compact self-adjoint linear operator. Therefore (see, for instance, Theorems VI.8 and VI.11 of [3]), there exists an orthonormal basis $\left\{w_{i}\right\}_{i=1}^{\infty}$ of $H$, consisting of eigenvectors of $\Lambda^{-1}$ such that

$$
\Lambda w_{i}=\lambda_{i} w_{i}, \quad \forall i \in \mathbb{N}
$$


with $\lambda_{i}>0$ and

$$
\lim _{i \rightarrow \infty} \lambda_{i}=+\infty
$$

Now,

$$
\left\|w_{i}\right\|_{V}^{2}=\left(w_{i}, w_{i}\right)_{V}=\left(\Lambda w_{i}, w_{i}\right)_{V^{\prime} \times V}=\left(\lambda_{i} w_{i}, w_{i}\right)_{H}=\lambda_{i} \quad \forall i=1, \cdots \infty .
$$

Therefore,

$$
u=\sum_{i=1}^{\infty} u_{i} w_{i} \in V \Leftrightarrow\|u\|_{V}^{2}=\sum_{i=1}^{\infty} \lambda_{i}\left|u_{i}\right|^{2}<\infty
$$

Furthermore, if $u=\sum_{i=1}^{\infty} u_{i} w_{i} \in V$ and $v=\sum_{i=1}^{\infty} v_{i} w_{i} \in V$, then

$$
(u, v)_{V}=\sum_{i=1}^{\infty} \lambda_{i} u_{i} v_{i}
$$

Definition 6 For every $r \in \mathbb{R}$ we define the Hilbert space $V^{r}$ in the following way:

$$
u=\sum_{i=1}^{\infty} u_{i} w_{i} \in V^{r} \Leftrightarrow\|u\|_{V^{r}}^{2}=\sum_{i=1}^{\infty} \lambda_{i}^{r}\left|u_{i}\right|^{2}<\infty .
$$

Furthermore, if $u=\sum_{i=1}^{\infty} u_{i} w_{i} \in V^{r}$ and $v=\sum_{i=1}^{\infty} v_{i} w_{i} \in V^{r}$, then

$$
(u, v)_{V^{r}}=\sum_{i=1}^{\infty} \lambda_{i}^{r} u_{i} v_{i}
$$

Remark 7 We have that $H=V^{0}, V=V^{1}$ and $V^{\prime}=V^{-1}$. Furthermore, $\left\{\lambda_{i}^{-r / 2} w_{i}\right\}_{i=1}^{\infty}$ is an orthonormal basis of $V^{r}$, for all $r \in \mathbb{R}$.

Definition 8 Let $\mathcal{H}=\left\{\sum_{\text {finite }} \mu_{j} w_{j}, \mu_{j} \in \mathbb{R}\right\}$ and $<w_{j}>=\left\{\mu w_{j}, \mu \in \mathbb{R}\right\}$ for all $j \in \mathbb{N}$. Given $i, j \in \mathbb{N}$, we consider the operator $w_{i} \otimes w_{j}: \mathcal{H} \rightarrow<w_{j}>$ defined by

$$
w_{i} \otimes w_{j}(\varphi)=\left(w_{i}, \varphi\right)_{H} w_{j} \quad \forall \varphi \in \mathcal{H}
$$

Now, since $\mathcal{H}$ is a dense subset of $V^{r}$, for all $r \in \mathbb{R}$, we can consider the extension $w_{i} \otimes w_{j}: V^{r} \rightarrow<w_{j}>$ defined by

$$
w_{i} \otimes w_{j}(\varphi)=\varphi_{i} w_{j} \quad \forall \varphi=\sum_{k=1}^{\infty} \varphi_{k} w_{k} \in V^{r}
$$


Remark 9 From Definition 2 it is easy to see that, for all $i, j \in \mathbb{N}$, we have that $w_{i} \otimes w_{j} \in V^{r} \widehat{\otimes}_{2} V^{s}$ for all $r, s \in \mathbb{R}$. Furthermore, if $P=\sum_{i, j=1}^{\infty} \xi_{i j} w_{i} \otimes w_{j}$, then

$$
P \in V^{r} \widehat{\otimes}_{2} V^{s} \Leftrightarrow\|P\|_{V^{r} \widehat{\otimes}_{2} V^{s}}^{2}=\sum_{i=1}^{\infty}\left\|P\left(\lambda_{i}^{-r / 2} w_{i}\right)\right\|_{V^{s}}^{2}<\infty
$$

Now,

$$
P\left(\lambda_{i}^{-r / 2} w_{i}\right)=\sum_{k, j=1}^{\infty} \xi_{k j}\left(w_{k}, \lambda_{i}^{-r / 2} w_{i}\right)_{H} w_{j}=\sum_{j=1}^{\infty} \xi_{i j} \lambda_{i}^{-r / 2} w_{j}
$$

Therefore

$$
\left\|P\left(\lambda_{i}^{-r / 2} w_{i}\right)\right\|_{V^{s}}^{2}=\sum_{j=1}^{\infty} \xi_{i j}^{2} \lambda_{i}^{-r} \lambda_{j}^{s}
$$

and we deduce that

$$
P \in V^{r} \widehat{\otimes}_{2} V^{s} \Leftrightarrow\|P\|_{V^{r} \widehat{\otimes}_{2} V^{s}}^{2}=\sum_{i, j=1}^{\infty} \xi_{i j}^{2} \lambda_{i}^{-r} \lambda_{j}^{s}<\infty
$$

Finally, if $Q=\sum_{i, j=1}^{\infty} \eta_{i j} w_{i} \otimes w_{j} \in V^{r} \widehat{\otimes}_{2} V^{s}$, then

$$
[P, Q]_{V^{r} \widehat{\otimes}_{2} V^{s}}=\sum_{i=1}^{\infty}\left(P\left(\lambda_{i}^{-r / 2} w_{i}\right), Q\left(\lambda_{i}^{-r / 2} w_{i}\right)\right)_{V^{s}}=\sum_{i, j=1}^{\infty} \xi_{i j} \eta_{i j} \lambda_{i}^{-r} \lambda_{j}^{s} .
$$

Example 10 Let $P=\sum_{i, j=1}^{\infty} \xi_{i j} w_{i} \otimes w_{j}$ and $Q=\sum_{i, j=1}^{\infty} \eta_{i j} w_{i} \otimes w_{j}$.

1. $P \in H \widehat{\otimes}_{2} H \Leftrightarrow\|P\|_{H \widehat{\otimes}_{2} H}^{2}=\sum_{i, j=1}^{\infty} \xi_{i j}^{2}<\infty$ and $[P, Q]_{H \widehat{\otimes}_{2} H}=\sum_{i, j=1}^{\infty} \xi_{i j} \eta_{i j}$.

2. $P \in V^{\prime} \widehat{\otimes}_{2} H \Leftrightarrow\|P\|_{V^{\prime} \widehat{\otimes}_{2} H}^{2}=\sum_{i, j=1}^{\infty} \xi_{i j}^{2} \lambda_{i}<\infty$ and $[P, Q]_{V^{\prime} \widehat{\otimes}_{2} H}=\sum_{i, j=1}^{\infty} \xi_{i j} \eta_{i j} \lambda_{i}$.

3. $P \in V \widehat{\otimes}_{2} H \Leftrightarrow\|P\|_{V \widehat{\otimes}_{2} H}^{2}=\sum_{i, j=1}^{\infty} \xi_{i j}^{2} \lambda_{i}^{-1}<\infty$ and $[P, Q]_{V \widehat{\otimes}_{2} H}=\sum_{i, j=1}^{\infty} \xi_{i j} \eta_{i j} \lambda_{i}^{-1}$.

4. $P \in H \widehat{\otimes}_{2} V \Leftrightarrow\|P\|_{H \widehat{\otimes}_{2} V}^{2}=\sum_{i, j=1}^{\infty} \xi_{i j}^{2} \lambda_{j}<\infty$ and $[P, Q]_{H \widehat{\otimes}_{2} V}=\sum_{i, j=1}^{\infty} \xi_{i j} \eta_{i j} \lambda_{j}$.

5. $P \in H \widehat{\otimes}_{2} V^{\prime} \Leftrightarrow\|P\|_{H \widehat{\otimes}_{2} V^{\prime}}^{2}=\sum_{i, j=1}^{\infty} \xi_{i j}^{2} \lambda_{j}^{-1}<\infty$ and $[P, Q]_{H \widehat{\otimes}_{2} V^{\prime}}=\sum_{i, j=1}^{\infty} \xi_{i j} \eta_{i j} \lambda_{j}^{-1}$. 


\subsection{Our particular case}

In this work we shall consider the spaces $V^{r} \widehat{\otimes}_{2} V^{s}, r, s \in \mathbb{R}$, for the particular case of $H=L^{2}(\mathcal{O})$ and $V=H_{0}^{1}(\mathcal{O}), \Lambda$ being now $-\Delta$ which is an isomorphism from $V$ to $V^{\prime}$. Therefore, we consider as orthonormal basis of $H$ the set $\left\{w_{1}, \ldots, w_{n}, ..\right\}$

$$
\begin{cases}-\Delta_{y} w_{n}=\lambda_{n} w_{n} & \text { in } \mathcal{O} \\ w_{n}=0 & \text { in } \partial \mathcal{O},\end{cases}
$$

with $\left\|w_{i}\right\|_{L^{2}(\mathcal{O})}=1$, for all $i=1,2, \cdots$. It has the the following properties:

$$
\left\{\begin{array}{l}
(a)\left(w_{n}, w_{m}\right)_{L^{2}(\mathcal{O})}=\delta_{n, m} \forall m, n . \\
(b)\left(w_{n}, w_{m}\right)_{H_{0}^{1}(\mathcal{O})}=\int_{\mathcal{O}} \nabla_{y} w_{n}(y) \nabla_{y} w_{m}(y) \mathrm{d} y=\lambda_{n} \delta_{n, m} \\
(c)\left\{\sum_{\text {finite }} \mu_{j} w_{j}, \mu_{j} \in \mathbb{R}\right\} \text { is a dense subset of } V .
\end{array}\right.
$$

If we write the sequence $\left\{\lambda_{1}, \ldots, \lambda_{n}, ..\right\}$ in a nondecreasing way, it can be proved (see, for instance, Theorem VI.8 of [3]) that $\lambda_{i} \geq 0$, for all $i=1, \cdots,+\infty$ and

$$
\lim _{i \rightarrow \infty} \lambda_{i}=+\infty
$$

Proposition 11 The identity operator $I: H \rightarrow H$ can be expressed as

$$
I=\sum_{i=1}^{\infty} w_{i} \otimes w_{i}
$$

with this series converging in $\mathcal{L}(H, H)$.

Proof. Let $\varphi \in H$, then

$$
\sum_{i=1}^{\infty} w_{i} \otimes w_{i}(\varphi)=\sum_{i=1}^{\infty}\left(\varphi, w_{i}\right)_{H} w_{i}=\sum_{i=1}^{\infty} \varphi_{i} w_{i}=\varphi
$$

where $\varphi_{i}, i=1, \cdots, \infty$ are the coordinates with respect to the orthonormal basis $\left\{w_{i}\right\}_{i=1}^{\infty}$ in $L^{2}(\mathcal{O})$.

Now the following question arises: Is the identity operator in some of the HilbertSchmidt spaces described above (as $H \widehat{\otimes}_{2} H$ or $V \widehat{\otimes}_{2} H$ )?

It is clear that $I \notin H \widehat{\otimes}_{2} H$, since (see Remark 9)

$$
\sum_{i=1}^{\infty} 1=\infty
$$

To see if the identity operator $I$ is in some of the other Hilbert-Schmidt spaces we shall use the following lemma. 
Lemma 12 (Weyl's Estimate) Given a regular domain $\Omega \subset \mathbb{R}^{n}$, the asymptotic behavior of the eigenvalues for the Laplace operator with homogeneous Dirichlet boundary conditions is the following:

$$
\lambda_{k} \sim \frac{4 \pi}{(\operatorname{Vol}(\Omega))^{2 / n}} k^{2 / n}
$$

Proof. See [14].

We point out that $w_{i} \in V^{s}$ for all $s \in \mathbb{R}$ and $i \in \mathbb{N}$. Further, $V^{0}=H$ and $V^{1}=V$. Depending on the domain dimension, we shall need to work on slightly more regular spaces than $H$ and $V$.

Definition 13 Let $\delta=0$ if $\operatorname{dim}(\mathcal{O})=1$ and $\delta>0$ if $\operatorname{dim}(\mathcal{O})=2(\operatorname{dim}(\mathcal{O})>2$ is not of interest in applications). Let us define $\widetilde{H}=V^{\delta}$ and $\widetilde{V}=V^{1+\delta}$ (if $\operatorname{dim}(\mathcal{O})=1$, then $\widetilde{H}=H$ and $\widetilde{V}=V)$. For $\delta \geq 0$ small enough, we have $\widetilde{V} \subset V \subset \widetilde{H} \subset H$.

Corollary 14 If $\operatorname{dim}(\mathcal{O}) \leq 2$, then $I \in\left(\widetilde{V} \widehat{\otimes}_{2} H\right) \cap\left(H \widehat{\otimes}_{2} \widetilde{V}^{\prime}\right)$.

Proof. We have to prove (see Remark 9) that

$$
\sum_{i=1}^{\infty} \frac{1}{\lambda_{i}^{1+\delta}}<+\infty
$$

which is a consequence of Lemma 12.

\section{A direct Study of the Riccati Initial Value Problem}

In [13], the author study a general equation

$$
\left\{\begin{array}{l}
\frac{\mathrm{d} P}{\mathrm{~d} x}+P A+A^{*} P+\Phi(P)=F \\
P(0)=P_{0}
\end{array}\right.
$$

with $A: V \rightarrow V^{\prime}$ coercive and $F \in L^{2}\left(0, a ; H \widehat{\otimes}_{2} H\right)$. Is is showed the existence of a solution $u \in \mathcal{C}\left([0, a]: H \widehat{\otimes}_{2} H\right)$. Let us study the Riccati equation

$$
\left\{\begin{array}{l}
\frac{\mathrm{d} P}{\mathrm{~d} x}-\alpha P \Delta_{y} P=\alpha^{-1} I \quad \text { a.e. for } x \in(0, a) \\
P(0)=0 .
\end{array}\right.
$$

In problem (4), we do not have a coercive operator $A$ and we have seen that $\alpha^{-1} I \notin H \widehat{\otimes}_{2} H$. We only have $\alpha^{-1} I \in\left(\widetilde{V} \widehat{\otimes}_{2} H\right) \cap\left(H \widehat{\otimes}_{2} \widetilde{V}^{\prime}\right)$ at least if $\operatorname{dim}(\mathcal{O}) \leq 2$. Furthermore $-\Delta_{y}$ is unbounded on $H$, so results of [13] cannot be applied directly. We shall try to get a solution $P \in \mathcal{C}\left([0, \infty): V^{r} \widehat{\otimes}_{2} V^{s}\right)$, for some $r, s \in \mathbb{R}$ to be determined, by a semi-discretization and passing to the limit process. 


\subsection{Semi discretization}

\subsubsection{Formulation of the problem and monotonicity results}

We approximate any space $V^{r}$, with $r \in \mathbb{R}$ by $\mathcal{V}^{m}=\operatorname{span}\left(w_{1}, \cdots, w_{m}\right)$ and define the $m$-approximate solution of (4) by the finite dimensional operator

$$
P^{m}(x)=\sum_{i, j=1}^{m} \xi_{i j}^{m}(x) w_{i} \otimes w_{j} \in \mathcal{V}^{m} \widehat{\otimes}_{2} \mathcal{V}^{m}
$$

where $\xi_{i j}^{m}(x)$ are chosen such that

$$
\left\{\begin{array}{l}
{\left[\frac{\mathrm{d} P^{m}}{\mathrm{~d} x}-\alpha P^{m} \Delta_{y} P^{m}-\alpha^{-1} I^{m}, w_{i} \otimes w_{j}\right]_{H \widehat{\otimes}_{2} H}=0, \forall i, j \in\{1, \cdots m\}} \\
P^{m}(0)=0
\end{array}\right.
$$

with $I^{m}=\sum_{i=1}^{m} w_{i} \otimes w_{i}$. We point out that $\xi_{i j}^{m}(x)=\left(P^{m}(x) w_{i}, w_{j}\right)_{H}$.

System (5) is a nonlinear system of equations in $\xi_{i j}^{m}$ of the form:

$$
\left\{\begin{array}{l}
\frac{\mathrm{d} \xi^{m}}{\mathrm{~d} x}+A^{m}\left(\xi^{m}\right)+b^{m}=0 \\
\xi^{m}(0)=0
\end{array}\right.
$$

where

$$
A^{m}\left(\xi^{m}\right)_{i j}(x)=-\alpha(x)\left[\left(\sum_{k, l=1}^{m} \xi_{k l}^{m} w_{k} \otimes w_{l}\right) \Delta_{y}\left(\sum_{r, s=1}^{m} \xi_{r s}^{m} w_{r} \otimes w_{s}\right), w_{i} \otimes w_{j}\right]_{H \widehat{\otimes}_{2} H}
$$

and

$$
b_{i j}^{m}(x)= \begin{cases}-\alpha^{-1}(x), & \text { if } i=j \\ 0, & \text { otherwise }\end{cases}
$$

It is easy to verify that

$$
A^{m}\left(\xi^{m}\right)_{i j}=-\alpha\left[\left(\sum_{k, l=1}^{m} \xi_{k l}^{m} w_{k} \otimes w_{l}\right)\left(\sum_{r, s=1}^{m}-\lambda_{s} \xi_{r s}^{m} w_{r} \otimes w_{s}\right), w_{i} \otimes w_{j}\right]_{H \widehat{\otimes}_{2} H} .
$$

Now, taking into account that

$$
w_{k} \otimes w_{l} \circ w_{r} \otimes w_{s}= \begin{cases}w_{r} \otimes w_{l}, & \text { if } k=s, \\ 0, & \text { otherwise }\end{cases}
$$

we have that

$$
A^{m}\left(\xi^{m}\right)_{i j}=\alpha\left[\sum_{k, l, r=1}^{m} \lambda_{k} \xi_{k l}^{m} \xi_{r k}^{m} w_{r} \otimes w_{l}, w_{i} \otimes w_{j}\right]_{H \widehat{\otimes}_{2} H}=\alpha \sum_{k=1}^{m} \lambda_{k} \xi_{i k}^{m} \xi_{k j}^{m}
$$


Then, if we write the $(i, j)$-coordinates in $m \times m$ matrix form, we obtain

$$
\left\{\begin{array}{l}
\frac{\mathrm{d} \xi^{m}}{\mathrm{~d} x}+\alpha \xi^{m} \Lambda^{m} \xi^{m}-\alpha^{-1} I^{m}=0 \\
\xi^{m}(0)=0
\end{array}\right.
$$

where $I^{m}$ represents here the $m \times m$ identity matrix and $\Lambda^{m}$ is a diagonal matrix, with $\lambda_{i}, i=1, \cdots m$, being the elements of the diagonal.

But this derivation is still formal since we have assumed the derivability of $P^{m}$. Then, by the theory of ordinary differential equations (Carathéodory Theorem), we know that there exists a unique local solution $P^{m}$ to $(5)$ in $[0, \delta]$, with $\delta$ small enough, which is absolutely continuous from $[0, \delta]$ to $\mathcal{V}^{m} \widehat{\otimes}_{2} \mathcal{V}^{m}$ (see, for instance, Theorems 5.1 and 5.3 of pages 28 and 30 of [7]). If $\alpha \in \mathcal{C}([0, a])$ we have $P^{m} \in \mathcal{C}^{1}\left([0, \delta]: \mathcal{V}^{m} \widehat{\otimes}_{2} \mathcal{V}^{m}\right)$.

The uniqueness of solution is also a consequence of Corollary 2 of page 13 of [12].

To go further, we need estimates on $\xi^{m}(s)$ (i.e. on $P^{m}(s)$ ) independent of $s$, in order to be able to extend the solution to $[0, \infty$ ) (see, for instance, Theorem 5.2 of page 29 of $[7])$.

Following page 11 of [12], the solution of the above problem can be expressed as

$$
\xi^{m}(x)=V(x) U(x)^{-1}
$$

where $Y=\left(\begin{array}{c}U \\ V\end{array}\right)$ satisfies the corresponding linear (Hamiltonian) matrix differential system

$$
\left\{\begin{array}{l}
\mathcal{J} Y^{\prime}(x)+\mathcal{U}(x) Y(x)=0 \\
Y(0)=\left(\begin{array}{c}
I^{m} \\
0
\end{array}\right)
\end{array}\right.
$$

with

$$
\mathcal{J}=\left(\begin{array}{cc}
0 & -I^{m} \\
I^{m} & 0
\end{array}\right), \quad \mathcal{U}(x)=\left(\begin{array}{cc}
\alpha^{-1}(x) I^{m} & 0 \\
0 & -\alpha(x) \Lambda^{m}
\end{array}\right)
$$

In order to study the global existence of solution, let us suppose first that $\alpha \in \mathbb{R}$ is a constant. Therefore,

$$
Y(x)=e^{A x}\left(\begin{array}{c}
I^{m} \\
0
\end{array}\right), \quad \forall x \in[0,+\infty)
$$

with

$$
A=-\mathcal{J}^{-1} \mathcal{U}=\left(\begin{array}{cc}
0 & -I^{m} \\
I^{m} & 0
\end{array}\right)\left(\begin{array}{cc}
\alpha^{-1} I^{m} & 0 \\
0 & -\alpha \Lambda^{m}
\end{array}\right)=\left(\begin{array}{cc}
0 & \alpha \Lambda^{m} \\
\alpha^{-1} I^{m} & 0
\end{array}\right)
$$

The eigenvalues of $A$ are $\sqrt{\lambda_{i}}$ and $-\sqrt{\lambda_{i}}, i=1, \cdots m$, and the matrix of eigenvectors is

$$
\left(\begin{array}{cc}
\alpha\left(\Lambda^{m}\right)^{1 / 2} & -\alpha\left(\Lambda^{m}\right)^{1 / 2} \\
I^{m} & I^{m}
\end{array}\right)
$$


Then $U$ and $V$ are given by

$$
\begin{array}{r}
U(x)=\frac{1}{2}\left(e^{\left(\Lambda^{m}\right)^{1 / 2} x}+e^{-\left(\Lambda^{m}\right)^{1 / 2} x}\right), \\
V(x)=\frac{\left(\Lambda^{m}\right)^{-1 / 2}}{2 \alpha}\left(e^{\left(\Lambda^{m}\right)^{1 / 2} x}-e^{-\left(\Lambda^{m}\right)^{1 / 2} x}\right) .
\end{array}
$$

Thus, $U$ is non-singular and we can extend the solution of (5) (when $\alpha$ is constant) from $[0, \delta]$ to $[0,+\infty]$.

Remark 15 The matrix $A$ has positive and negative eigenvalues. The initial value problem for the Hamiltonian system (7) is well posed in finite dimension. This would not be the case for the corresponding infinite dimensional initial value problem because of the part of system (7) corresponding to positive eigenvalues $\left\{\sqrt{\lambda_{i}}\right\}_{i=0}^{\infty}$ which are going to $+\infty$. For this part of the system we have a situation similar to a backward heat equation whose ill-posedness is well-known.

Let us consider

$$
\underline{P}^{m}(x)=\sum_{i, j=1}^{m} \underline{\xi}_{i j}^{m}(x) w_{i} \otimes w_{j} \in \mathcal{V}^{m} \widehat{\otimes}_{2} \mathcal{V}^{m}
$$

and

$$
\bar{P}^{m}(x)=\sum_{i, j=1}^{m} \bar{\xi}_{i j}^{m}(x) w_{i} \otimes w_{j} \in \mathcal{V}^{m} \widehat{\otimes}_{2} \mathcal{V}^{m},
$$

the particular cases of $\alpha \equiv \underline{\alpha}$ and $\alpha \equiv \bar{\alpha}$ respectively. We have proved above that $\underline{P}^{m}$ and $\bar{P}^{m}$ are defined over $[0,+\infty]$.

Proposition 16 For a given $m \in \mathbb{N}$, we have that, for all $x \geq 0$,

$$
\begin{aligned}
& \underline{P}^{m}(x)=\sum_{i=1}^{m} \underline{\xi}_{i i}^{m}(x) w_{i} \otimes w_{i}, \\
& \bar{P}^{m}(x)=\sum_{i=1}^{m} \bar{\xi}_{i i}^{m}(x) w_{i} \otimes w_{i},
\end{aligned}
$$

and there exists $\underline{P}_{\infty}^{m}, \bar{P}_{\infty}^{m} \in \mathcal{V}^{m} \widehat{\otimes}_{2} \mathcal{V}^{m}$ such that, if $0 \leq t_{1} \leq t_{2}$, then

$$
\begin{aligned}
& 0 \leq \underline{P}^{m}\left(t_{1}\right) \leq \underline{P}^{m}\left(t_{2}\right) \leq \underline{P}_{\infty}^{m}, \\
& 0 \leq \bar{P}^{m}\left(t_{1}\right) \leq \bar{P}^{m}\left(t_{2}\right) \leq \bar{P}_{\infty}^{m},
\end{aligned}
$$

Proposition 17 For a given $m \in \mathbb{N}$, we can extend the solution $P^{m}$ of (5) (with $\alpha$ not necessarily constant) from $[0, \delta]$ to $[0, a]$. Further, for all $x \in[0, a]$,

$$
P^{m}(x)=\sum_{i=1}^{m} \xi_{i i}^{m}(x) w_{i} \otimes w_{i} \in W^{1, \infty}\left(0, \delta: \mathcal{V}^{m} \widehat{\otimes}_{2} \mathcal{V}^{m}\right),
$$

and for all $x \in[0, a]$ we have that $0 \leq \underline{P}^{m}(x) \leq P^{m}(x) \leq \bar{P}^{m}(x)$. 


\subsubsection{Proof of Propositions 16 and 17}

\section{Proof Proposition 16}

From (8)-(9) it is easy to see that $\underline{P}^{m}$ and $\bar{P}^{m}$ are diagonal and given by

$$
\underline{\xi}_{i i}^{m}(x)=\frac{1}{\underline{\alpha} \sqrt{\lambda_{i}}} \tanh \left(\sqrt{\lambda_{i}} x\right), \quad i=1, \ldots, m
$$

and

$$
\bar{\xi}_{i i}^{m}(x)=\frac{1}{\bar{\alpha} \sqrt{\lambda_{i}}} \tanh \left(\sqrt{\lambda_{i}} x\right), \quad i=1, \ldots m
$$

They can be also computed as the solution of the diagonal systems

$$
\begin{aligned}
& \left\{\begin{array}{l}
\frac{\mathrm{d} \underline{\xi}_{i i}^{m}}{\mathrm{~d} x}+\underline{\alpha} \lambda_{i}\left(\underline{\xi}_{i i}^{m}\right)^{2}-\underline{\alpha}^{-1}=0, \\
\underline{\xi}_{i i}^{m}(0)=0,
\end{array}\right. \\
& \left\{\begin{array}{l}
\frac{\mathrm{d} \bar{\xi}_{i i}}{\mathrm{~d} x}+\bar{\alpha} \lambda_{i}\left(\bar{\xi}_{i i}^{m}\right)^{2}-\bar{\alpha}^{-1}=0, \\
\bar{\xi}_{i i}^{m}(0)=0,
\end{array}\right.
\end{aligned}
$$

Now,

$$
\begin{aligned}
& \frac{\mathrm{d} \underline{\xi}_{i i}^{m}}{\mathrm{~d} x}(x)=\frac{\underline{\alpha}^{-1}}{\cosh ^{2}\left(\sqrt{\lambda_{i}} x\right)}>0, \quad \forall x \in \mathbb{R}, \\
& \frac{\mathrm{d} \bar{\xi}_{i i}^{m}}{\mathrm{~d} x}(x)=\frac{\bar{\alpha}^{-1}}{\cosh ^{2}\left(\sqrt{\lambda_{i}} x\right)}>0, \quad \forall x \in \mathbb{R},
\end{aligned}
$$

and we can define

$$
\underline{\xi}_{\infty, i i}^{m}:=\lim _{x \rightarrow \infty} \underline{\xi}_{i i}^{m}(x)=\frac{1}{\underline{\alpha} \sqrt{\lambda_{i}}}, \quad i=1, \ldots, m
$$

and

$$
\bar{\xi}_{\infty, i i}^{m}:=\lim _{x \rightarrow \infty} \bar{\xi}_{i i}^{m}(x)=\frac{1}{\bar{\alpha} \sqrt{\lambda_{i}}}, \quad i=1, \ldots, m,
$$

which concludes the proof.

\section{Proof of Proposition 17}

The unique solution of (5) is the same as the solution of the diagonal system

$$
\left\{\begin{array}{l}
\frac{\mathrm{d} \xi_{i i}^{m}}{\mathrm{~d} x}+\alpha(x) \lambda_{i}\left(\xi_{i i}^{m}\right)^{2}-\alpha^{-1}(x)=0, \quad i=1, \ldots, m . \\
\xi_{i i}^{m}(0)=0
\end{array}\right.
$$

Further, since

$$
\underline{\alpha}^{-1}-\underline{\alpha} \lambda_{i} \xi^{2} \leq \alpha^{-1}(x)-\alpha(x) \lambda_{i} \xi^{2} \leq \bar{\alpha}^{-1}-\bar{\alpha} \lambda_{i} \xi^{2}, \quad \forall \xi \in \mathbb{R},
$$


we have that (see, for instance, Theorem 6.1 of page 51 of [7])

$$
\underline{\xi}_{i i}^{m}(x) \leq \xi_{i i}^{m}(x) \leq \bar{\xi}_{i i}^{m}(x), \quad \forall x \in[0, a]
$$

which concludes the proof by applying, for instance, the continuation Theorem 5.2 of page 29 of [7].

In Figure 1 we show the graphs of $\xi_{i i}^{m}(x), \underline{\xi}_{i i}^{m}(x)$ and $\bar{\xi}_{i i}^{m}(x)$ for the case of $\lambda_{i}=1$ and $\alpha(x)=5.5+4.5 \sin (2 \pi x)$ (which implies that $\underline{\alpha}=10$ and $\bar{\alpha}=1$ ).

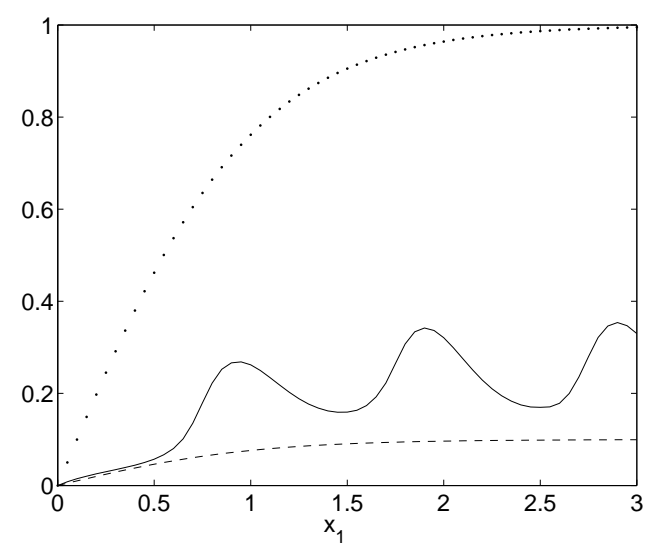

Figure 1: Graph of $\xi_{i i}^{m}(-), \underline{\xi}_{i i}^{m}(--)$ and $\bar{\xi}_{i i}^{m}(\cdots)$ for the case of $\lambda_{i}=1$ and $\alpha(x)=5.5+4.5 \sin (2 \pi x)$ (which implies that $\underline{\alpha}=10$ and $\bar{\alpha}=1$ ).

Remark 18 From (10) and (11) we obtain that

$$
0 \leq \underline{\alpha} \lambda_{i}\left(\underline{\xi}_{i i}^{m}\right)^{2}(x)<\underline{\alpha}^{-1} \text { and } 0<\frac{\mathrm{d} \underline{\xi}_{i i}^{m}}{\mathrm{~d} x}(x) \leq \underline{\alpha}^{-1},
$$

and

$$
0 \leq \bar{\alpha} \lambda_{i}\left(\bar{\xi}_{i i}^{m}\right)^{2}(x)<\bar{\alpha}^{-1} \text { and } 0<\frac{\mathrm{d} \bar{\xi}_{i i}^{m}}{\mathrm{~d} x}(x) \leq \bar{\alpha}^{-1},
$$

for all $x \in \mathbb{R}, i=1, \ldots, \infty$. Therefore,

$$
0 \leq \alpha(x) \lambda_{i}\left(\xi_{i i}^{m}\right)^{2}(x)<\alpha(x) \bar{\alpha}^{-2}
$$

and

$$
\underline{\alpha}^{-1}-\underline{\alpha} \bar{\alpha}^{-2}=\underline{\alpha}^{-1}-\underline{\alpha} \lambda_{i}\left(\bar{\xi}_{\infty, i i}^{m}\right)^{2} \leq \underline{\alpha}^{-1}-\underline{\alpha} \lambda_{i}\left(\bar{\xi}_{i i}^{m}\right)^{2} \leq \frac{\mathrm{d} \xi_{i i}}{\mathrm{~d} x}(x) \leq \bar{\alpha}^{-1},
$$

for all $x \in \mathbb{R}, i=1, \ldots, \infty$. 


\subsection{Passing to the limit. Existence of solution. Regularity and Monotonicity Results}

Definition 19 An operator $T \in \mathcal{L}(H, H)$ is said to be positive $(T \geq 0)$ if $(T v, v)_{H} \geq 0$ for all $v \in H$.

Remark 20 If $T \in \mathcal{L}(H, H)$ satisfies $T_{i j}=\left(T w_{i}, w_{j}\right)=0$ if $i \neq j$, then $T$ is said to be diagonal and $T \geq 0$ if and only if $T_{i i} \geq 0$ for all $i=1,2, \cdots$.

We can now define the solution of the Riccati equation (4) in a Hilbert-Schmidt framework.

Theorem $21 \underline{P}^{m}$ and $\bar{P}^{m}$ have a limit $\underline{P}$ and $\bar{P}$ respectively in $\mathcal{C}\left([0, \infty): \widetilde{H} \widehat{\otimes}_{2} H \cap\right.$ $\left.H \widehat{\otimes}_{2} \widetilde{H}^{\prime}\right) \cap \mathcal{C}^{1}\left([0, \infty): \widetilde{V} \widehat{\otimes}_{2} H \cap H \widehat{\otimes}_{2} \widetilde{V}^{\prime}\right)$ as $m \rightarrow \infty$. Furthermore $\underline{P}, \bar{P} \in \mathcal{C}([0,+\infty)$ : $\left.\mathcal{L}\left(V^{s}, V^{s+1}\right)\right) \cap \mathcal{C}^{1}\left([0,+\infty): \mathcal{L}\left(V^{s}, V^{s}\right)\right)$, for all $s \in \mathbb{R}$ and there exist $\underline{P}_{\infty}, \bar{P}_{\infty} \in \widetilde{H}_{\otimes_{2}} H \cap$ $H \widehat{\otimes}_{2} \widetilde{H}^{\prime}$ such that, if $0 \leq t_{1} \leq t_{2}$, then

$$
0 \leq \underline{P}\left(t_{1}\right) \leq \underline{P}\left(t_{2}\right) \leq \underline{P}_{\infty}
$$

and

$$
0 \leq \bar{P}\left(t_{1}\right) \leq \bar{P}\left(t_{2}\right) \leq \bar{P}_{\infty}
$$

Proof. Let us do the proof for $\underline{P}^{m}$ (the other case is analogous). The proof is based on the passing to the limit of $\underline{P}^{m}$ and of the differential equation satisfied by $\underline{P}^{m}$ as $m \rightarrow \infty$. Now,

$$
\underline{P}_{\infty}^{m}=\sum_{i=1}^{m} \frac{1}{\underline{\alpha} \sqrt{\lambda_{i}}} w_{i} \otimes w_{i} .
$$

Therefore, if $0<m_{1} \leq m_{2}$, then

$$
0 \leq \underline{P}_{\infty}^{m_{1}} \leq \underline{P}_{\infty}^{m_{2}} \quad \text { and } \quad\left(\underline{P}_{\infty}^{m_{1}}\right)_{i i}=\left(\underline{P}_{\infty}^{m_{2}}\right)_{i i} \quad \text { for } i=1, \cdots m_{1} .
$$

Further,

$$
\underline{P}_{\infty}=\sum_{i=1}^{\infty} \frac{1}{\underline{\alpha} \sqrt{\lambda_{i}}} w_{i} \otimes w_{i} \in \widetilde{H} \widehat{\otimes}_{2} H \cap H \widehat{\otimes}_{2} \widetilde{H}^{\prime}
$$

since

$$
\left\|\underline{P}_{\infty}\right\|_{\widetilde{H} \widehat{\otimes}_{2} H}^{2}=\left\|\underline{P}_{\infty}\right\|_{H \widehat{\otimes}_{2} \widetilde{H}^{\prime}}^{2}=\frac{1}{\underline{\alpha}} \sum_{i=1}^{\infty} \frac{1}{\lambda_{i}^{1+\delta}}<+\infty .
$$

Then, $\underline{P}_{\infty}^{m} \rightarrow \underline{P}_{\infty}$ in the topology of $\widetilde{H} \widehat{\otimes}_{2} H \cap H \widehat{\otimes}_{2} \widetilde{H}^{\prime}$.

Now, since we are dealing with diagonal matrices, it is easy to prove that, if $0<m_{1} \leq$ $m_{2}$, then $\underline{\xi}_{i i}^{m_{1}}(x)=\underline{\xi}_{i i}^{m_{2}}(x)$ for $i=1, \cdots, m_{1}$ and $0 \leq \underline{P}^{m_{1}}(x) \leq \underline{P}^{m_{2}}(x) \leq \underline{P}_{\infty}$, which implies that, if we define $\underline{\xi}_{i i}=\underline{\xi}_{i i}^{i}$, then

$$
\underline{P}^{m} \stackrel{m \rightarrow \infty}{\longrightarrow} \underline{P}=\sum_{i=1}^{\infty} \underline{\xi}_{i i} w_{i} \otimes w_{i} \quad \text { in } \mathcal{C}\left([0, \infty): \widetilde{H} \widehat{\otimes}_{2} H \cap H \widehat{\otimes}_{2} \widetilde{H}^{\prime}\right),
$$


since, using (13),

$$
\begin{aligned}
& \left\|\underline{P}^{m}-\underline{P}\right\|_{\mathcal{C}\left([0, \infty): \widetilde{H} \widehat{\otimes}_{2} H\right)}^{2}=\left\|\underline{P}^{m}-\underline{P}\right\|_{\mathcal{C}\left([0, \infty): H \widehat{\otimes}_{2} \widetilde{H}^{\prime}\right)}^{2} \\
& =\sup _{x \in[0, \infty)} \sum_{i=m+1}^{\infty} \frac{\left|\underline{\xi}_{i i}(x)\right|^{2}}{\lambda_{i}^{\delta}} \leq \underline{\alpha}^{-2} \sum_{i=m+1}^{\infty} \frac{1}{\lambda_{i}^{1+\delta}} \stackrel{m \rightarrow \infty}{\longrightarrow} 0 .
\end{aligned}
$$

Now (13) gives the expected regularity on $\underline{P}$ because

$$
\|\underline{P}\|_{\mathcal{C}\left([0, \infty): \mathcal{L}\left(V^{s}, V^{s+1}\right)\right)}^{2}=\sup _{x \in[0, \infty)} \max _{i} \lambda_{i}\left|\underline{\xi}_{i i}(x)\right|^{2} \leq \underline{\alpha}^{-2} .
$$

Further, if $0 \leq t_{1} \leq t_{2}$, then

$$
0 \leq \underline{P}\left(t_{1}\right) \leq \underline{P}\left(t_{2}\right) \leq \underline{P}_{\infty}
$$

We also claim that

$$
\frac{\mathrm{d} \underline{P}^{m}}{\mathrm{~d} x} \stackrel{m \rightarrow \infty}{\longrightarrow} \frac{\mathrm{d} \underline{P}}{\mathrm{~d} x} \quad \text { in } \mathcal{C}\left([0, \infty): \widetilde{V} \widehat{\otimes}_{2} H \cap H \widehat{\otimes}_{2} \tilde{V}^{\prime}\right),
$$

since, from (13),

$$
\begin{gathered}
\left\|\frac{\mathrm{d} \underline{P}^{m}}{\mathrm{~d} x}-\frac{\mathrm{d} \underline{P}}{\mathrm{~d} x}\right\|_{\mathcal{C}\left([0, \infty): \widetilde{V} \widehat{\otimes}_{2} H\right)}^{2}=\left\|\frac{\mathrm{d} \underline{P}^{m}}{\mathrm{~d} x}-\frac{\mathrm{d} \underline{P}}{\mathrm{~d} x}\right\|_{\mathcal{C}\left([0, \infty): H \widehat{\otimes}_{2} \widetilde{V}^{\prime}\right)}^{2} \\
=\sup _{x \in[0, \infty)} \sum_{i=m+1}^{\infty} \frac{1}{\lambda_{i}^{1+\delta}}\left|\frac{\mathrm{d} \underline{\xi}_{i i}}{\mathrm{~d} x}(x)\right|^{2} \\
=\sup _{x \in[0, \infty)} \sum_{i=m+1}^{\infty} \frac{\left|\underline{\alpha}^{-1}-\underline{\alpha} \lambda_{i}\left(\underline{\xi}_{i i}(x)\right)^{2}\right|^{2}}{\lambda_{i}^{1+\delta}} \\
\leq \sum_{i=m+1}^{\infty} \frac{\underline{\alpha}^{-2}}{\lambda_{i}^{1+\delta}} \stackrel{m \rightarrow \infty}{\longrightarrow} 0 .
\end{gathered}
$$

From (13) again

$$
\left\|\frac{\mathrm{d} \underline{P}}{\mathrm{~d} x}\right\|_{\mathcal{C}\left([0,+\infty): \mathcal{L}\left(V^{s}, V^{s}\right)\right)}^{2}=\sup _{x \in[0, \infty)} \max _{i}\left|\underline{\alpha}^{-1}-\underline{\alpha} \lambda_{i}\left(\underline{\xi}_{i i}(x)\right)^{2}\right|^{2} \leq \underline{\alpha}^{-2} .
$$

Similarly, it is easy to deduce that

$$
\underline{\alpha}^{-1} I^{m} \stackrel{m \rightarrow \infty}{\longrightarrow} \underline{\alpha}^{-1} I \quad \text { in } \mathcal{C}\left([0, \infty): \widetilde{V} \widehat{\otimes}_{2} H \cap H \widehat{\otimes}_{2} \widetilde{V}^{\prime}\right)
$$

and

$$
\underline{\alpha} \underline{P}^{m} \Delta_{y} \underline{P}^{m} \stackrel{m \rightarrow \infty}{\longrightarrow} \underline{\alpha} \underline{P} \Delta_{y} \underline{P} \quad \text { in } \mathcal{C}\left([0, \infty): \widetilde{V} \widehat{\otimes}_{2} H \cap H \widehat{\otimes}_{2} \widetilde{V}^{\prime}\right) .
$$

Then $\underline{P} \in \mathcal{C}\left([0, \infty): \widetilde{H} \widehat{\otimes}_{2} H \cap H \widehat{\otimes}_{2} \widetilde{H}^{\prime}\right) \cap \mathcal{C}^{1}\left([0, \infty): \widetilde{V} \widehat{\otimes}_{2} H \cap H \widehat{\otimes}_{2} \widetilde{V}^{\prime}\right)$ satisfies

$$
\frac{\mathrm{d} \underline{P}}{\mathrm{~d} x}-\underline{\alpha} \underline{P} \Delta_{y} \underline{P}-\underline{\alpha}^{-1} I=0,
$$

where each term is considered as an element of $\widetilde{V} \widehat{\otimes}_{2} H \cap H \widehat{\otimes}_{2} \widetilde{V}^{\prime}$. 
Remark 22 There exists a constant $C>0$, independent of $s$, such that

$$
\|\underline{P}\|_{\mathcal{C}\left([0,+\infty): \mathcal{L}\left(V^{s}, V^{s+1}\right)\right)}+\|\underline{P}\|_{\mathcal{C}^{1}\left([0,+\infty): \mathcal{L}\left(V^{s}, V^{s}\right)\right)} \leq C
$$

and

$$
\|\bar{P}\|_{\mathcal{C}\left([0,+\infty): \mathcal{L}\left(V^{s}, V^{s+1}\right)\right)}+\|\bar{P}\|_{\mathcal{C}^{1}\left([0,+\infty): \mathcal{L}\left(V^{s}, V^{s}\right)\right)} \leq C .
$$

Theorem 23 Problem (4) has a solution $P$ limit of $P^{m}$ in $\mathcal{C}\left([0, a]: \widetilde{H} \widehat{\otimes}_{2} H \cap H \widehat{\otimes}_{2} \widetilde{H}^{\prime}\right) \cap$ $W^{1, \infty}\left(0, a: \widetilde{V} \widehat{\otimes}_{2} H \cap H \widehat{\otimes}_{2} \widetilde{V}^{\prime}\right)$ as $m \rightarrow \infty$. Furthermore $P \in \mathcal{C}\left([0, a]: \mathcal{L}\left(V^{s}, V^{s+1}\right)\right) \cap$ $W^{1, \infty}\left(0, a: \mathcal{L}\left(V^{s}, V^{s}\right)\right)$, for all $s \in \mathbb{R}$ and

$$
0 \leq \underline{P}(x) \leq P(x) \leq \bar{P}(x) .
$$

Proof. Since we are dealing with diagonal matrices, it is easy to prove that, if $0<m_{1} \leq$ $m_{2}$, then $\xi_{i i}^{m_{1}}(x)=\xi_{i i}^{m_{2}}(x)$ for $i=1, \cdots, m_{1}$ and $0 \leq P^{m}(x) \leq \bar{P}_{\infty}$, for all $m \in \mathbb{N}$, which implies that, if we define $\xi_{i i}=\xi_{i i}^{i}$, then

$$
P^{m} \stackrel{m \rightarrow \infty}{\longrightarrow} P=\sum_{i=1}^{\infty} \xi_{i i} w_{i} \otimes w_{i} \quad \text { in } \mathcal{C}\left([0, a]: \widetilde{H} \widehat{\otimes}_{2} H \cap H \widehat{\otimes}_{2} \widetilde{H}^{\prime}\right)
$$

since, using (15),

$$
\begin{aligned}
& \left\|P^{m}-P\right\|_{\mathcal{C}\left([0, a]: \widetilde{H} \widehat{\otimes}_{2} H\right)}^{2}=\left\|P^{m}-P\right\|_{\mathcal{C}\left([0, a]: H \widehat{\otimes}_{2} \widetilde{H}^{\prime}\right)}^{2} \\
& =\sup _{x \in[0, a]} \sum_{i=m+1}^{\infty} \frac{\left|\xi_{i i}(x)\right|^{2}}{\lambda_{i}^{\delta}} \leq \bar{\alpha}^{-2} \sum_{i=m+1}^{\infty} \frac{1}{\lambda_{i}^{1+\delta}} \stackrel{m \rightarrow \infty}{\longrightarrow} 0 .
\end{aligned}
$$

Now (15) gives the expected regularity on $P$ because

$$
\|P\|_{\mathcal{C}\left([0, a]: \mathcal{L}\left(V^{s}, V^{s+1}\right)\right)}^{2}=\sup _{x \in[0, a]} \max _{i} \lambda_{i}\left|\xi_{i i}(x)\right|^{2} \leq \bar{\alpha}^{-2} .
$$

Further, if $x \in[0, a]$, then

$$
0 \leq \underline{P}(x) \leq P(x) \leq \bar{P}(x) \leq P_{\infty} .
$$

We also claim that

$$
\frac{\mathrm{d} P^{m}}{\mathrm{~d} x} \stackrel{m \rightarrow \infty}{\longrightarrow} \frac{\mathrm{d} P}{\mathrm{~d} x} \quad \text { in } L^{\infty}\left(0, a: \tilde{V} \widehat{\otimes}_{2} H \cap H \widehat{\otimes}_{2} \widetilde{V}^{\prime}\right)
$$

since, from (16),

$$
\begin{aligned}
\left\|\frac{\mathrm{d} P^{m}}{\mathrm{~d} x}-\frac{\mathrm{d} P}{\mathrm{~d} x}\right\|_{L^{\infty}\left(0, a: \widetilde{V} \widehat{\otimes}_{2} H\right)}^{2}=\left\|\frac{\mathrm{d} P^{m}}{\mathrm{~d} x}-\frac{\mathrm{d} P}{\mathrm{~d} x}\right\|_{L^{\infty}\left(0, a: H \widehat{\otimes}_{2} \widetilde{V}^{\prime}\right)}^{2} \\
=\underset{x \in(0, a)}{\operatorname{ess} \sup _{i=m+1}} \sum_{i=m}^{\infty} \frac{1}{\lambda_{i}^{1+\delta}}\left|\frac{\mathrm{d} \xi_{i i}}{\mathrm{~d} x}(x)\right|^{2}
\end{aligned}
$$




$$
\begin{aligned}
& =\operatorname{esssup}_{x \in(0, a)} \sum_{i=m+1}^{\infty} \frac{\left|\alpha(x)^{-1}-\alpha(x) \lambda_{i}\left(\xi_{i i}(x)\right)^{2}\right|^{2}}{\lambda_{i}^{1+\delta}} \\
& \leq \sum_{i=m+1}^{\infty} \max \left\{\bar{\alpha}^{-2},\left(\underline{\alpha} \lambda_{i} \bar{\xi}_{i i, \infty}^{m}-\underline{\alpha}^{-1}\right)^{2}\right\} \frac{1}{\lambda_{i}^{1+\delta}} \\
& \leq \max \left\{\bar{\alpha}^{-2}, \lambda_{1} \underline{\alpha}^{2} \bar{\alpha}^{-2}\right\} \sum_{i=m+1}^{\infty} \frac{1}{\lambda_{i}^{1+\delta}} \stackrel{m \rightarrow \infty}{\longrightarrow} 0 .
\end{aligned}
$$

From (16) again

$$
\begin{aligned}
\left\|\frac{\mathrm{d} P}{\mathrm{~d} x}\right\|_{L^{\infty}\left(0, a: \mathcal{L}\left(V^{s}, V^{s}\right)\right)}^{2}= & \operatorname{ess}_{x \in(0, a)} \max _{i}\left|\alpha(x)^{-1}-\alpha(x) \lambda_{i}\left(\xi_{i i}(x)\right)^{2}\right|^{2} \\
& \leq \max \left\{\bar{\alpha}^{-2}, \lambda_{1} \underline{\alpha}^{2} \bar{\alpha}^{-2}\right\} .
\end{aligned}
$$

Similarly, it is easy to deduce that

$$
\alpha^{-1} I^{m} \stackrel{m \rightarrow \infty}{\longrightarrow} \alpha^{-1} I \quad \text { in } L^{\infty}\left(0, a: \widetilde{V} \widehat{\otimes}_{2} H \cap H \widehat{\otimes}_{2} \widetilde{V}^{\prime}\right)
$$

and

$$
\alpha P^{m} \Delta_{y} P^{m} \stackrel{m \rightarrow \infty}{\longrightarrow} \alpha P \Delta_{y} P \quad \text { in } L^{\infty}\left(0, a: \widetilde{V} \widehat{\otimes}_{2} H \cap H \widehat{\otimes}_{2} \widetilde{V}^{\prime}\right) .
$$

Then $P \in \mathcal{C}\left([0, a]: \widetilde{H} \widehat{\otimes}_{2} H \cap H \widehat{\otimes}_{2} \widetilde{H}^{\prime}\right) \cap W^{1, \infty}\left(0, a: \widetilde{V} \widehat{\otimes}_{2} H \cap H \widehat{\otimes}_{2} \widetilde{V}^{\prime}\right)$ satisfies

$$
\frac{\mathrm{d} P}{\mathrm{~d} x}-\alpha P \Delta_{y} P-\alpha^{-1} I=0,
$$

where each term is considered as an element of $\widetilde{V} \widehat{\otimes}_{2} H \cap H \widehat{\otimes}_{2} \widetilde{V}^{\prime}$.

Remark 24 There exists a constant $C>0$, independent of $s$, such that

$$
\|P\|_{\mathcal{C}\left([0, a]: \mathcal{L}\left(V^{s}, V^{s+1}\right)\right)}+\|P\|_{W^{1, \infty}\left(0, a: \mathcal{L}\left(V^{s}, V^{s}\right)\right)} \leq C .
$$

Remark 25 If $\alpha \in \mathcal{C}([0, a])$ then

$$
P^{m} \stackrel{m \rightarrow \infty}{\longrightarrow} P \text { in } \mathcal{C}\left([0, a]: \widetilde{H} \widehat{\otimes}_{2} H \cap H \widehat{\otimes}_{2} \widetilde{H}^{\prime}\right) \cap \mathcal{C}^{1}\left([0, a]: \widetilde{V} \widehat{\otimes}_{2} H \cap H \widehat{\otimes}_{2} \widetilde{V}^{\prime}\right)
$$

and $P \in \mathcal{C}\left([0, a]: \mathcal{L}\left(V^{s}, V^{s+1}\right)\right) \cap \mathcal{C}^{1}\left([0, a]: \mathcal{L}\left(V^{s}, V^{s}\right)\right)$, for all $s \in \mathbb{R}$. Furthermore, there exists a constant $C>0$, independent of $s$, such that

$$
\|P\|_{\mathcal{C}\left([0, a]: \mathcal{L}\left(V^{s}, V^{s+1}\right)\right)}+\|P\|_{\mathcal{C}^{1}\left([0, a]: \mathcal{L}\left(V^{s}, V^{s}\right)\right)} \leq C .
$$




\subsection{Uniqueness of Solution}

Lemma 26 Suppose $T$ is a symmetric operator satisfying $T \in \mathcal{L}\left(V^{p}, V^{p+r}\right)$ for some $r \in \mathbb{R}$ and for all $p \geq k(k \in \mathbb{R})$ and there exists a constant $C>0$ independent of $p$ such that $\|T\|_{\mathcal{L}\left(V^{p}, V^{p+r}\right)}^{2} \leq C$, then $T$ is diagonal, i.e., $T_{i, j}=\left(T w_{i}, w_{j}\right)_{H}=0$ if $i \neq j$.

\section{Proof.}

$$
\begin{aligned}
\|T\|_{\mathcal{L}\left(V^{p}, V^{p+r}\right)}^{2} & \geq \sup _{i}\left\|T \frac{w_{i}}{\left\|w_{i}\right\|_{V^{p}}}\right\|_{V^{p+r}}^{2}=\sup _{i}\left\|\lambda_{i}^{-p / 2} T w_{i}\right\|_{V^{p+r}}^{2} \\
& =\sup _{i}\left\|\lambda_{i}^{-p / 2} \sum_{j=1}^{\infty} T_{i, j} w_{j}\right\|_{V^{p+r}}^{2}=\sup _{i} \lambda_{i}^{-p} \sum_{j=1}^{\infty} \lambda_{j}^{p+r}\left|T_{i, j}\right|^{2}
\end{aligned}
$$

Therefore, $T_{i, j}=0$ if $i \neq j$ and $\|T\|_{\mathcal{L}\left(V^{p}, V^{p+r}\right)}^{2} \geq \sup _{i} \lambda_{i}^{r}\left|T_{i, i}\right|^{2}$, because, otherwise, since

$$
\lambda_{i}^{-p} \lambda_{j}^{p} \rightarrow \infty \text { as } p \rightarrow \infty\left(\text { if } \lambda_{j}>\lambda_{i}\right)
$$

we would have

$$
\|T\|_{\mathcal{L}\left(V^{p}, V^{p+r}\right)}^{2} \rightarrow \infty \text { as } p \rightarrow \infty
$$

in contradiction with the assumptions.

Theorem 27 The solution $P$ of (4) is unique in the set of functions

$$
\begin{aligned}
& \left\{P \in W^{1, \infty}\left(0, a ; V^{p} \widehat{\otimes}_{2} V^{q}\right) \text { for some } p, q \in \mathbb{R}:\right. \\
& \qquad \begin{aligned}
P \in \mathcal{C}([0,+\infty): & \left.\mathcal{L}\left(V^{s}, V^{s+1}\right)\right) \text { for all } s \in \mathbb{R}, \\
& \text { with } \left.\|P\|_{\mathcal{C}\left([0,+\infty): \mathcal{L}\left(V^{s}, V^{s+1}\right)\right)}^{2} \text { uniformly bounded in } s\right\} .
\end{aligned}
\end{aligned}
$$

Proof. Let $P$ a solution of (4) in the above class of functions. From Theorem $26 P$ is diagonal. Let

$$
P(x)=\sum_{i=1}^{\infty} \xi_{i i}(x) w_{i} \otimes w_{i} \in W^{1, \infty}\left(0, a ; V^{p} \widehat{\otimes}_{2} V^{q}\right)
$$

be the Fourier expansion of $P(x)$. Then, equation (4) can be written as

$$
\sum_{i=1}^{\infty}\left(\frac{\mathrm{d} \xi_{i i}}{\mathrm{~d} x}(x)+\alpha(x) \lambda_{i} \xi_{i i}^{2}\right) w_{i} \otimes w_{i}=\sum_{i=1}^{\infty} \alpha(x)^{-1} w_{i} \otimes w_{i},
$$

with both terms of the above equality in $\left(\widetilde{V} \widehat{\otimes}_{2} H\right) \cap\left(H \widehat{\otimes}_{2} \widetilde{V}^{\prime}\right)$. Then, by the uniqueness of the Fourier representation, we have that

$$
\frac{\mathrm{d} \xi_{i i}}{\mathrm{~d} x}(x)+\alpha(x) \lambda_{i} \xi_{i i}^{2}=\alpha(x)^{-1}
$$

Therefore, if $\xi^{m}$ is the matrix given by $\xi^{m}=\left(\xi_{i j}\right)_{i, j=1}^{m}$, then $\xi^{m}$ is the unique solution of (6) (as we proved in Section 4.1.1), which concludes the proof due to the strong convergence of $\xi^{m}$ to $\xi$ in $\mathcal{C}\left([0, a]: \widetilde{H} \widehat{\otimes}_{2} H \cap H \widehat{\otimes}_{2} \widetilde{H}^{\prime}\right)$ proved in Theorem 23. 


\section{Elliptic problems associated to the Riccati Equa- tion (4)}

In this section we show how the Hilbert-Schmidt operator $P$ solution of the Riccati equation (4) can be used to transform the second order elliptic boundary value problem (1) into one or two uncoupled first order initial value problems (system (2)).

\section{$5.1 \quad$ Function $r$}

Theorem 28 Let $s \in \mathbb{R}$ arbitrary and $u_{0} \in V^{s}$. Then, there exists a unique solution $r \in \mathcal{C}\left([0, a]: V^{s}\right) \cap L^{2}\left(0, a: V^{s+1 / 2}\right) \cap H^{1}\left(0, a: V^{s-1 / 2}\right)$, of the initial value problem

$$
\left\{\begin{array}{l}
\frac{\mathrm{d} r}{\mathrm{~d} x}-\alpha P \Delta_{y} r=0 \\
r(0)=u_{0}
\end{array}\right.
$$

Proof. Let

$$
u_{0}=\sum_{i=1}^{\infty} u_{0 i} w_{i}
$$

be the Fourier decomposition of $u_{0}$. Then, the solution of the problem is given by

$$
r(x, y)=\sum_{i=1}^{\infty} r_{i}(x) w_{i}(y),
$$

where each Fourier coefficient $r_{i}(x), i=1, \cdots, \infty$, satisfies the differential equation

$$
\left\{\begin{array}{l}
\frac{\mathrm{d} r_{i}}{\mathrm{~d} x}=-\alpha \lambda_{i} \xi_{i i} r_{i}, \\
r_{i}(0)=u_{0 i} .
\end{array}\right.
$$

Then,

$$
r_{i}(x)=u_{0 i} e^{-\int_{0}^{x} \lambda_{i} \alpha(t) \xi_{i i}(t) \mathrm{d} t} \in \mathcal{C}([0, a])
$$

and

$$
\begin{aligned}
\left|r_{i}(x)\right| & \leq\left|u_{0 i}\right| e^{-\bar{\alpha} \lambda_{i} \int_{0}^{x} \underline{\xi}_{i i}(t) \mathrm{d} t}=\left|u_{0 i}\right| e^{-\frac{\bar{\alpha} \lambda_{i}}{\underline{\alpha} \sqrt{\lambda_{i}}} \int_{0}^{x} \frac{\sinh \left(\sqrt{\lambda_{i}} t\right)}{\cosh \left(\sqrt{\lambda_{i}} t\right)} \mathrm{d} t} \\
& =\frac{\left|u_{0 i}\right|}{\left(\cosh \sqrt{\lambda_{i}} x\right)^{\bar{\alpha}} \underline{\alpha}^{-1}} \leq\left|u_{0 i}\right| .
\end{aligned}
$$

Now, for all $x \in[0, a]$, we have that

$$
\sum_{i=1}^{\infty} \lambda_{i}^{s}\left|r_{i}(x)\right|^{2} \leq \sum_{i=1}^{\infty} \lambda_{i}^{s}\left|u_{0 i}\right|^{2}<\infty
$$


which implies that $r(x) \in V^{s}$. Hence, since $r_{i} \in \mathcal{C}([0, a]), i=1, \cdots, \infty$, and thanks to the uniform convergence of the previous series, we have that $r \in \mathcal{C}\left([0, a]: V^{s}\right)$. To obtain the $L^{2}\left(0, a: V^{s+1 / 2}\right)$ regularity consider

$$
\begin{aligned}
\int_{0}^{a} \sum_{i=1}^{\infty} \lambda_{i}^{s+1 / 2}\left|r_{i}(x)\right|^{2} \mathrm{~d} x & \leq 2^{2 \bar{\alpha} \underline{\alpha}^{-1}} \sum_{i=1}^{\infty} \lambda_{i}^{s}\left|u_{0 i}\right|^{2} \int_{0}^{a} \frac{\sqrt{\lambda_{i}}}{e^{2 \bar{\alpha} \underline{\alpha}-1 \sqrt{\lambda_{i}} x}} \mathrm{~d} x \\
& =\frac{2^{2 \bar{\alpha} \underline{\alpha}^{-1}}}{2 \bar{\alpha} \underline{\alpha}^{-1}} \sum_{i=1}^{\infty}\left(1-e^{-2 \bar{\alpha} \underline{\alpha}^{-1} \sqrt{\lambda_{i}} a}\right) \lambda_{i}^{s}\left|u_{0 i}\right|^{2}<\infty
\end{aligned}
$$

To obtain the $H^{1}\left(0, a: V^{s-1 / 2}\right)$ regularity consider

$$
\begin{gathered}
\int_{0}^{a} \sum_{i=1}^{\infty} \lambda_{i}^{s-1 / 2}\left|\frac{\mathrm{d} r_{i}}{\mathrm{~d} x}(x)\right|^{2} \mathrm{~d} x \leq 2^{2 \bar{\alpha} \underline{\alpha}^{-1}} \underline{\alpha}^{2} \bar{\alpha}^{-2} \sum_{i=1}^{\infty} \lambda_{i}^{s}\left|u_{0 i}\right|^{2} \int_{0}^{a} \frac{\sqrt{\lambda_{i}}}{e^{2 \bar{\alpha} \underline{\alpha}-1 \sqrt{\lambda_{i}} x}} \mathrm{~d} x \\
=2^{\left(2 \bar{\alpha} \underline{\alpha}^{-1}-1\right)} \underline{\alpha}^{3} \bar{\alpha}^{-3} \sum_{i=1}^{\infty}\left(1-e^{-2 \bar{\alpha} \underline{\alpha}^{-1} \sqrt{\lambda_{i}} a}\right) \lambda_{i}^{s}\left|u_{0 i}\right|^{2}<\infty .
\end{gathered}
$$

Remark 29 In order to prove that $r \in \mathcal{C}\left([0, a]: V^{s}\right)$ we could have also used the theory of intermediate spaces (see, for instance, [10]), which gives

$$
L^{2}\left(0, a: V^{s+1 / 2}\right) \cap H^{1}\left(0, a: V^{s-1 / 2}\right) \subset \mathcal{C}\left([0, a]:\left[V^{s-1 / 2}, V^{s+1 / 2}\right]_{1 / 2}\right)
$$

and $\left[V^{s-1 / 2}, V^{s+1 / 2}\right]_{1 / 2}=V^{s}$.

Proposition 30 If $\alpha \in \mathcal{C}([0, a])$ then $\left.\left.r \in \mathcal{C}^{1}\left([0, a]: V^{s-1}\right) \cap \mathcal{C}^{1}(] 0, a\right]: V^{p}\right)$, for any $p \in \mathbb{N}$.

Proof. Let us prove now that $r \in \mathcal{C}^{1}\left([0, a]: V^{s-1}\right)$. We have

$$
\left|\frac{\mathrm{d} r_{i}}{\mathrm{~d} x}(x)\right| \leq \underline{\alpha} \bar{\alpha}^{-1} \sqrt{\lambda_{i}} \frac{\sinh \left(\sqrt{\lambda_{i}} x\right)}{\left(\cosh \left(\sqrt{\lambda_{i}} x\right)\right)^{1+\bar{\alpha} \underline{\alpha}^{-1}}}\left|u_{0 i}\right| \leq \underline{\alpha} \bar{\alpha}^{-1} \sqrt{\lambda_{i}}\left|u_{0 i}\right|
$$

which implies that, for all $x \in[0, a]$,

$$
\sum_{i=1}^{\infty} \lambda_{i}^{s-1}\left|\frac{\mathrm{d} r_{i}}{\mathrm{~d} x}(x)\right|^{2} \leq \underline{\alpha}^{2} \bar{\alpha}^{-2} \sum_{i=1}^{\infty} \lambda_{i}^{s}\left|u_{0 i}\right|^{2}<\infty
$$

Thus, $\frac{\mathrm{d} r}{\mathrm{~d} x}(x, \cdot) \in V^{s-1}$, for all $x \in[0, a]$, and therefore $r \in \mathcal{C}^{1}\left([0, a]: V^{s-1}\right)$.

To prove the additional regularity we consider

$$
\sum_{i=1}^{\infty} \lambda_{i}^{p}\left|r_{i}(x)\right|^{2} \leq 2^{2 \bar{\alpha} \underline{\alpha}^{-1}} \sum_{i=1}^{\infty} \lambda_{i}^{s}\left|u_{0 i}\right|^{2} \lambda_{i}^{p-s} e^{-2 \bar{\alpha} \underline{\alpha}^{-1} \sqrt{\lambda_{i}} x}<\infty
$$

for any $x>0$, since for any $\mu, k>0$

$$
\lambda^{k} e^{-\mu \sqrt{\lambda}} \longrightarrow 0 \quad \text { as } \lambda \rightarrow+\infty \text {. }
$$

Similarly, it can be proved that $\left.\left.\frac{\mathrm{d} r}{\mathrm{~d} x} \in \mathcal{C}(] 0, a\right]: V^{p}\right)$. 
Theorem 31 Let $s \in \mathbb{R}$ arbitrary and $f \in L^{2}\left(0, a: V^{s}\right)$. Then, there exists a unique solution $r \in \mathcal{C}\left([0, a]: V^{s+3 / 2}\right) \cap L^{2}\left(0, a: V^{s+2}\right) \cap H^{1}\left(0, a: V^{s+1}\right)$, of the initial value problem

$$
\left\{\begin{array}{l}
\frac{\mathrm{d} r}{\mathrm{~d} x}-\alpha P \Delta_{y} r=P f \\
r(0)=0
\end{array}\right.
$$

Proof. Let

$$
f(x, \cdot)=\sum_{i=1}^{\infty} f_{i}(x) w_{i}
$$

be the Fourier decomposition of $f(x, \cdot)$, for all $x \in[0, a]$ (respectively, a.e. in $(0, a)$ ), with $f_{i} \in \mathcal{C}([0, a])$ (respectively, $\left.f_{i} \in L^{2}(0, a)\right)$. Then, the solution of the problem is given by

$$
r(x, y)=\sum_{i=1}^{\infty} r_{i}(x) w_{i}(y)
$$

where each Fourier coefficient $r_{i}(x), i=1, \cdots, \infty$, satisfies the differential equation

$$
\left\{\begin{array}{l}
\frac{\mathrm{d} r_{i}}{\mathrm{~d} x}=\xi_{i i}\left(-\alpha \lambda_{i} r_{i}+f_{i}\right) \\
r_{i}(0)=0
\end{array}\right.
$$

Then, multiplying in (17) by $r_{i}$ and integrating over $(0, x)$ we obtain

$$
\frac{1}{2}\left|r_{i}(x)\right|^{2}+\int_{0}^{x} \alpha(s) \lambda_{i} \xi_{i i}(s)\left|r_{i}(s)\right|^{2} \mathrm{~d} s=\int_{0}^{x} \xi_{i i}(s) f_{i}(s) r_{i}(s) \mathrm{d} s .
$$

Then

$$
\begin{gathered}
\frac{1}{2}\left|r_{i}(x)\right|^{2}+\int_{0}^{x} \alpha(s) \lambda_{i} \xi_{i i}(s)\left|r_{i}(s)\right|^{2} \mathrm{~d} s \\
\leq \frac{1}{2} \int_{0}^{x} \bar{\alpha} \lambda_{i} \xi_{i i}(s)\left|r_{i}(s)\right|^{2} \mathrm{~d} s+\frac{1}{2} \int_{0}^{x} \frac{1}{\bar{\alpha} \lambda_{i}} \xi_{i i}(s)\left|f_{i}(s)\right|^{2} \mathrm{~d} s
\end{gathered}
$$

and therefore, we obtain

$$
\left|r_{i}(x)\right|^{2} \leq \int_{0}^{x} \frac{1}{\bar{\alpha}^{2} \lambda_{i}^{1+1 / 2}}\left|f_{i}(s)\right|^{2} \mathrm{~d} s
$$

Thus,

$$
\sum_{i=1}^{\infty} \lambda_{i}^{s+3 / 2}\left|r_{i}(x)\right|^{2} \leq \frac{1}{\bar{\alpha}^{2}} \sum_{i=1}^{\infty} \lambda_{i}^{s}\left\|f_{i}\right\|_{L^{2}(0, a)}^{2}=\frac{1}{\bar{\alpha}^{2}}\|f\|_{L^{2}\left(0, a: V^{s}\right)}^{2}<\infty,
$$

which implies that $r(x) \in V^{s+3 / 2}$. Hence, since $r_{i} \in \mathcal{C}([0, a])$ (because $f_{i} \in L^{1}(0, a)$ ), $i=1, \cdots, \infty$, and thanks to the uniform convergence of the series we have that $r \in$ $\mathcal{C}\left([0, a]: V^{s+3 / 2}\right)$. 
To obtain the $L^{2}\left(0, a: V^{s+2}\right)$ regularity consider

$$
\begin{aligned}
\int_{0}^{a}\left|r_{i}(x)\right|^{2} \mathrm{~d} x & \leq \int_{0}^{a} \int_{0}^{x} \frac{1}{\bar{\alpha} \lambda_{i}} \xi_{i i}(s)\left|f_{i}(s)\right|^{2} \mathrm{~d} s \mathrm{~d} x \leq \int_{0}^{a} \frac{1}{\bar{\alpha} \lambda_{i}} \bar{\xi}_{i i}(x)\left\|f_{i}\right\|_{L^{2}(0, a)}^{2} \mathrm{~d} x \\
& =\left\|f_{i}\right\|_{L^{2}(0, a)}^{2} \frac{1}{\bar{\alpha}^{2} \lambda_{i}^{2}} \ln (\cosh a) .
\end{aligned}
$$

Therefore

$$
\begin{aligned}
\|r\|_{L^{2}\left(0, a: V^{s+2}\right)}^{2} & =\int_{0}^{a} \sum_{i=1}^{\infty} \lambda_{i}^{s+2}\left|r_{i}(x)\right|^{2} \mathrm{~d} x \leq \frac{\ln (\cosh a)}{\bar{\alpha}^{2}} \sum_{i=1}^{\infty} \lambda_{i}^{s}\left\|f_{i}\right\|_{L^{2}(0, a)}^{2} \\
& =\frac{\ln (\cosh a)}{\bar{\alpha}^{2}}\|f\|_{L^{2}\left(0, a: V^{s}\right)}^{2}<\infty
\end{aligned}
$$

Finally, since $\xi_{i i} \in L^{\infty}(0, a)$ is uniformly bounded and $f_{i} \in L^{2}(0, a)$, from (17) and Theorem 23 we have that $r_{i} \in H^{1}(0, a)$ and we deduce that $r \in H^{1}\left(0, a: V^{s+1}\right)$.

Remark 32 The solution $r_{i}$ of (17) is

$$
r_{i}(x)=e^{-\int_{0}^{x} \alpha(t) \lambda_{i} \xi_{i i}(t) \mathrm{d} t} \int_{0}^{x} \xi_{i i}(s) f_{i}(s) e^{\int_{0}^{s} \alpha(t) \lambda_{i} \xi_{i i}(t) \mathrm{d} t} \mathrm{~d} s .
$$

Remark 33 In order to prove that $r \in \mathcal{C}\left([0, a]: V^{s+3 / 2}\right)$ we could have also used the theory of intermediate spaces (see, for instance, $[10]$ ), which gives

$$
L^{2}\left(0, a: V^{s+2}\right) \cap H^{1}\left(0, a: V^{s+1}\right) \subset \mathcal{C}\left([0, a]:\left[V^{s+1}, V^{s+2}\right]_{1 / 2}\right)
$$

and $\left[V^{s+1}, V^{s+2}\right]_{1 / 2}=V^{s+3 / 2}$.

Remark 34 If $f \in L^{\infty}\left(0, a: V^{s}\right)$ then $r \in W^{1, \infty}\left(0, a: V^{s+1 / 2}\right)$. Furthermore, if $\alpha \in$ $\mathcal{C}([0, a])$ and $f \in \mathcal{C}\left([0, a]: V^{s}\right)$ then $r \in \mathcal{C}^{1}\left([0, a]: V^{s+1 / 2}\right)$.

Corollary 35 Let $j, k \in \mathbb{R}$ arbitrary, $u_{0} \in V^{j}$ and $f \in L^{2}\left(0, a: V^{k}\right)$. Then, if $p=$ $\min \{j, k+3 / 2\}$, there exists a unique solution $r \in \mathcal{C}\left([0, a]: V^{p}\right) \cap L^{2}\left(0, a: V^{p+1 / 2}\right) \cap$ $\left.H^{1}\left(0, a: V^{p-1 / 2}\right)\right)$, of the initial value problem

$$
\left\{\begin{array}{l}
\frac{\mathrm{d} r}{\mathrm{~d} x}-\alpha P \Delta_{y} r=P f \\
r(0)=u_{0}
\end{array}\right.
$$

Furthermore, each Fourier coefficient $r_{i}, i=1, \cdots, \infty$, is given by

$$
r_{i}(x)=e^{-\int_{0}^{x} \alpha(t) \lambda_{i} \xi_{i i}(t) \mathrm{d} t}\left(u_{0 i}+\int_{0}^{x} \xi_{i i}(s) f_{i}(s) e^{\int_{0}^{s} \alpha(t) \lambda_{i} \xi_{i i}(t) \mathrm{d} t} \mathrm{~d} s\right) .
$$

Remark 36 With the assumptions of the above Corollary, if $\alpha \in \mathcal{C}([0, a])$ and $f \in$ $\mathcal{C}\left([0, a]: V^{k}\right)$, then $r \in \mathcal{C}^{1}\left([0, a]: V^{p-1}\right)$. 


\subsection{Dirichlet Condition on $\Gamma_{a}$}

In the following Lemma 37 and Corollary 38 we give existence and uniqueness results for the corresponding solution $u$ in a suitable functional space which enables us to prove that $u$ is solution of a Poisson equation. Therefore we do not try to obtain all and/or the best regularity results, since they can be obtained later as a result of the well-known regularity results for the Poisson equation.

Lemma 37 Let $j, k \in \mathbb{R}$ arbitrary, $u_{0} \in V^{j}$ and $f \in L^{2}\left(0, a: V^{k}\right)$. Then, if $p=$ $\min \{j, k+3 / 2\}$, there exists a unique solution $u \in \mathcal{C}\left([0, a]: V^{p}\right)$ of the initial value problem

$$
\left\{\begin{array}{l}
P \alpha \frac{\mathrm{d} u}{\mathrm{~d} x}=u-r \\
u(a)=0
\end{array}\right.
$$

where $r \in \mathcal{C}\left([0, a]: V^{p}\right) \cap L^{2}\left(0, a: V^{p+1 / 2}\right) \cap H^{1}\left(0, a: V^{p-1 / 2}\right)$ is the solution of the initial value problem (19). Furthermore, $u=\sum_{i=1}^{\infty} u_{i} w_{i}$, with $u_{i} \in \mathcal{C}([0, a]), \frac{\mathrm{d} u_{i}}{\mathrm{~d} x} \in L^{\infty}(0, a)$ and $\alpha \frac{\mathrm{d} u_{i}}{\mathrm{~d} x} \in W^{1, \infty}(0, a)$, for all $i \in \mathbb{N}$.

Proof. The solution of the problem is given by

$$
u(x, y)=\sum_{i=1}^{\infty} u_{i}(x) w_{i}(y)
$$

where each Fourier coefficient $u_{i}(x), i=1, \cdots, \infty$, satisfies the differential equation

$$
\left\{\begin{array}{l}
\xi_{i i} \alpha \frac{\mathrm{d} u_{i}}{\mathrm{~d} x}=u_{i}-r_{i} \\
u_{i}(a)=0
\end{array}\right.
$$

Solving (21) we obtain

$$
\begin{gathered}
u_{i}(x)=e^{-\int_{x}^{a}\left(\alpha(t) \xi_{i i}(t)\right)^{-1} \mathrm{~d} t} \int_{x}^{a}\left(\alpha(s) \xi_{i i}(s)\right)^{-1} r_{i}(s) e^{\int_{s}^{a}\left(\alpha(t) \xi_{i i}(t)\right)^{-1} \mathrm{~d} t} \mathrm{~d} s \\
=e^{-\int_{x}^{a}\left(\alpha(t) \xi_{i i}(t)\right)^{-1} \mathrm{~d} t}\left(r_{i}(x) e^{\int_{x}^{a}\left(\alpha(t) \xi_{i i}(t)\right)^{-1} \mathrm{~d} t}+\int_{x}^{a}\left(\frac{\mathrm{d} r_{i}}{\mathrm{~d} x}(s) e^{\int_{s}^{a}\left(\alpha(t) \xi_{i i}(t)\right)^{-1} \mathrm{~d} t} \mathrm{~d} s\right)\right. \\
=r_{i}(x)+e^{-\int_{x}^{a}\left(\alpha(t) \xi_{i i}(t)\right)^{-1} \mathrm{~d} t} \int_{x}^{a}\left(\frac{\mathrm{d} r_{i}}{\mathrm{~d} x}(s) e^{\int_{s}^{a}\left(\alpha(t) \xi_{i i}(t)\right)^{-1} \mathrm{~d} t} \mathrm{~d} s .\right.
\end{gathered}
$$

Now $\xi_{i i}$ is 0 at $x=0$ and we have to check the behaviour of $u_{i}$ near $x=0$. Let us see that $u_{i} \in \mathcal{C}([0, a]), i=1, \cdots, \infty$. It is easy to see that

$$
e^{-\int_{x}^{a}\left(\alpha(t) \xi_{i i}(t)\right)^{-1} \mathrm{~d} t} \leq\left(\frac{\sinh \left(\sqrt{\lambda_{i}} x\right)}{\sinh \left(\sqrt{\lambda_{i}} a\right)}\right)^{\bar{\alpha} \underline{\alpha}^{-1}} \rightarrow 0 \quad \text { as } \quad x \rightarrow 0 .
$$


Now, since $r_{i} \in \mathcal{C}([0, a])$, it is easy to prove that, if $r_{i}(0)=u_{0 i} \neq 0$, then

$$
\lim _{x \rightarrow 0}\left|\int_{x}^{a}\left(\alpha(s) \xi_{i i}(s)\right)^{-1} r_{i}(s) e^{\int_{s}^{a}\left(\alpha(t) \xi_{i i}(t)\right)^{-1} \mathrm{~d} t} \mathrm{~d} s\right|=\infty .
$$

Therefore, if $u_{0 i}=0$ and (23) does not hold, then (obviously)

$$
\lim _{x \rightarrow 0} u_{i}(x)=0
$$

Otherwise, using L'Hôpital's rule,

$$
\begin{aligned}
\lim _{x \rightarrow 0} u_{i}(x) & =\lim _{x \rightarrow 0} \frac{\int_{x}^{a}\left(\alpha(s) \xi_{i i}(s)\right)^{-1} r_{i}(s) e^{\int_{s}^{a}\left(\alpha(t) \xi_{i i}(t)\right)^{-1} \mathrm{~d} t} \mathrm{~d} s}{e^{\int_{x}^{a}\left(\alpha(t) \xi_{i i}(t)\right)^{-1} \mathrm{~d} t}} \\
& =\lim _{x \rightarrow 0} \frac{\left(\alpha(x) \xi_{i i}(x)\right)^{-1} r_{i}(x) e^{\int_{x}^{a}\left(\alpha(t) \xi_{i i}(t)\right)^{-1} \mathrm{~d} t}}{\left(\alpha(x) \xi_{i i}(x)\right)^{-1} e^{\int_{x}^{a}\left(\alpha(t) \xi_{i i}(t)\right)^{-1} \mathrm{~d} t}}=r_{i}(0)=u_{0 i} .
\end{aligned}
$$

This implies that $u_{i} \in \mathcal{C}([0, a])$ for all $i=1, \cdots, \infty$. The results about $\frac{\mathrm{d} u_{i}}{\mathrm{~d} x}$ and $\alpha \frac{\mathrm{d} u_{i}}{\mathrm{~d} x}$ are easily verified.

Let us prove that $u \in \mathcal{C}\left([0, a]: V^{p}\right)$. From (21) we have

$$
\begin{gathered}
\int_{x}^{a} \xi_{i i}(s) \alpha(s)\left(\frac{\mathrm{d} u_{i}}{\mathrm{~d} x}(s)-\frac{\mathrm{d} r_{i}}{\mathrm{~d} x}(s)\right)^{2} \mathrm{~d} s \\
=\frac{1}{2} \int_{x}^{a} \frac{\mathrm{d}}{\mathrm{d} x}\left(u_{i}(s)-r_{i}(s)\right)^{2} \mathrm{~d} s-\int_{x}^{a} \xi_{i i}(s) \alpha(s) \frac{\mathrm{d} r_{i}}{\mathrm{~d} x}(s)\left(\frac{\mathrm{d} u_{i}}{\mathrm{~d} x}(s)-\frac{\mathrm{d} r_{i}}{\mathrm{~d} x}(s)\right) \mathrm{d} s \\
=-\frac{1}{2}\left(u_{i}(x)-r_{i}(x)\right)^{2}+\frac{1}{2}|r(a)|^{2}-\int_{x}^{a} \xi_{i i}(s) \alpha(s) \frac{\mathrm{d} r_{i}}{\mathrm{~d} x}(s)\left(\frac{\mathrm{d} u_{i}}{\mathrm{~d} x}(s)-\frac{\mathrm{d} r_{i}}{\mathrm{~d} x}(s)\right) \mathrm{d} s .
\end{gathered}
$$

Therefore,

$$
\begin{gathered}
\frac{1}{2}\left(u_{i}(x)-r_{i}(x)\right)^{2}+\int_{x}^{a} \xi_{i i}(s) \alpha(s)\left(\frac{\mathrm{d} u_{i}}{\mathrm{~d} x}(s)-\frac{\mathrm{d} r_{i}}{\mathrm{~d} x}(s)\right)^{2} \mathrm{~d} s \leq \\
\frac{1}{2}\left|r_{i}(a)\right|^{2}+\frac{1}{2} \int_{x}^{a} \xi_{i i}(s) \alpha(s)\left|\frac{\mathrm{d} r_{i}}{\mathrm{~d} x}(s)\right|^{2} \mathrm{~d} s+\frac{1}{2} \int_{x}^{a} \xi_{i i}(s) \alpha(s)\left(\frac{\mathrm{d} u_{i}}{\mathrm{~d} x}(s)-\frac{\mathrm{d} r_{i}}{\mathrm{~d} x}(s)\right)^{2} \mathrm{~d} s .
\end{gathered}
$$

Hence,

$$
\begin{gathered}
\left|u_{i}(x)\right|^{2}+\left|r_{i}(x)\right|^{2}+\int_{x}^{a} \xi_{i i}(s) \alpha(s)\left(\frac{\mathrm{d} u_{i}}{\mathrm{~d} x}(s)-\frac{\mathrm{d} r_{i}}{\mathrm{~d} x}(s)\right)^{2} \mathrm{~d} s \leq \\
\frac{\left|u_{i}(x)\right|^{2}}{2}+2\left|r_{i}(x)\right|^{2}+\left|r_{i}(a)\right|^{2}+\frac{\alpha}{\bar{\alpha}} \int_{0}^{a} \frac{1}{\sqrt{\lambda_{i}}}\left|\frac{\mathrm{d} r_{i}}{\mathrm{~d} x}(s)\right|^{2} \mathrm{~d} s,
\end{gathered}
$$


which implies that

$$
\sum_{i=1}^{\infty} \lambda_{i}^{p}\left|u_{i}(x)\right|^{2} \leq 2 \sum_{i=1}^{\infty} \lambda_{i}^{p}\left(\left|r_{i}(x)\right|^{2}+\left|r_{i}(a)\right|^{2}\right)+2 \frac{\underline{\alpha}}{\bar{\alpha}}\left\|\frac{\mathrm{d} r}{\mathrm{~d} x}\right\|_{H^{1}\left(0, a: V^{p-1 / 2}\right)}<\infty .
$$

Therefore $u(x, \cdot) \in V^{p}$ for all $x \in[0, a]$ and thanks to the uniform convergence of the series we have that $u \in \mathcal{C}\left([0, a]: V^{p}\right)$, since $u_{i} \in \mathcal{C}([0, a])$ and

$$
\begin{gathered}
\left\|u-\sum_{i=1}^{m} u_{i} w_{i}\right\|_{\mathcal{C}\left([0, a]: V^{p}\right)}=\sup _{x \in[0, a]} \sum_{i=m+1}^{\infty} \lambda_{i}^{p}\left|u_{i}(x)\right|^{2} \\
\leq 2 \sum_{i=m+1}^{\infty} \lambda_{i}^{p}\left|r_{i}(a)\right|^{2}+\frac{\underline{\alpha}}{\bar{\alpha}} \sum_{i=m+1}^{\infty} \lambda_{i}^{p-1 / 2} \int_{0}^{a}\left|\frac{\mathrm{d} r_{i}}{\mathrm{~d} x}(s)\right|^{2} \mathrm{~d} s \stackrel{m \rightarrow \infty}{\longrightarrow} 0 .
\end{gathered}
$$

Corollary 38 Let $j, k, q \in \mathbb{R}$ arbitrary, $u_{0} \in V^{j}, f \in L^{2}\left(0, a: V^{k}\right)$ and $u_{a} \in V^{q}$. Then, if $p=\min \{j, k+3 / 2, q\}$, there exists a unique solution $u \in \mathcal{C}\left([0, a]: V^{p}\right)$ of the initial value problem

$$
\left\{\begin{array}{l}
P \alpha \frac{\mathrm{d} u}{\mathrm{~d} x}=u-r \\
u(a)=u_{a}
\end{array}\right.
$$

where $r \in \mathcal{C}\left([0, a]: V^{\bar{p}}\right) \cap L^{2}\left(0, a: V^{\bar{p}+1 / 2}\right) \cap H^{1}\left(0, a: V^{\bar{p}-1 / 2}\right)$, is the solution of the initial value problem (19), with $\bar{p}=\min \{j, k+3 / 2\}$. Furthermore, each Fourier coefficient $u_{i}$, $i=1, \cdots, \infty$, satisfies $u_{i} \in \mathcal{C}([0, a]), \frac{\mathrm{d} u_{i}}{\mathrm{~d} x} \in L^{\infty}(0, a), \alpha \frac{\mathrm{d} u_{i}}{\mathrm{~d} x} \in W^{1, \infty}(0, a)$ and

$$
\begin{aligned}
u_{i}(x)= & e^{-\int_{x}^{a}\left(\alpha(t) \xi_{i i}(t)\right)^{-1} \mathrm{~d} t} \\
& \left(u_{a i}+\int_{x}^{a}\left(\alpha(s) \xi_{i i}(s)\right)^{-1} r_{i}(s) e^{\int_{s}^{a}\left(\alpha(t) \xi_{i i}(t)\right)^{-1} \mathrm{~d} t} \mathrm{~d} s\right) .
\end{aligned}
$$

Theorem 39 Let $j, k, q \in \mathbb{R}$ arbitrary, $u_{0} \in V^{j}, f \in L^{2}\left(0, a: V^{k}\right)$ and $u_{a} \in V^{q}$. Then, if $p=\min \{j, k+3 / 2, q\}$, the unique solution $u \in \mathcal{C}\left([0, a]: V^{p}\right)$ of the initial value problem (24) is also the unique solution of the elliptic problem

$$
\begin{cases}-\nabla \cdot(\alpha(x) \nabla u)=f & \text { in } \Omega, \\ u=0 & \text { on } \Sigma, \\ u=u_{0} & \text { on } \Gamma_{0}, \\ u=u_{a} & \text { on } \Gamma_{a} .\end{cases}
$$

Proof. Derivating (21) with respect to the $x$ variable we get

$$
\frac{\mathrm{d} u_{i}}{\mathrm{~d} x}-\frac{\mathrm{d} \xi_{i i}}{\mathrm{~d} x} \alpha \frac{\mathrm{d} u_{i}}{\mathrm{~d} x}-\frac{\mathrm{d} r_{i}}{\mathrm{~d} x}=\xi_{i i} \frac{\mathrm{d}}{\mathrm{d} x}\left(\alpha \frac{\mathrm{d} u_{i}}{\mathrm{~d} x}\right),
$$

which is an equation with all its terms in $L^{\infty}(0, a)$ (see Theorem 23 and regularity results of Corollary 38). Then, taking into account the Riccati equation satisfied by $\xi_{i i}$ and the equation satisfied by $r_{i}$, we have that

$$
\xi_{i i} \frac{\mathrm{d}}{\mathrm{d} x}\left(\alpha \frac{\mathrm{d} u_{i}}{\mathrm{~d} x}\right)=\alpha^{2} \lambda_{i} \xi_{i i}^{2} \frac{\mathrm{d} u_{i}}{\mathrm{~d} x}+\xi_{i i}\left(\alpha \lambda_{i} r_{i}-f_{i}\right) .
$$


Therefore, we have the following equation in $L^{2}(0, a)$ :

$$
\xi_{i i}\left[\frac{\mathrm{d}}{\mathrm{d} x}\left(\alpha \frac{\mathrm{d} u_{i}}{\mathrm{~d} x}\right)\right]=\xi_{i i}\left[\alpha \lambda_{i} u_{i}-f_{i}\right]
$$

Hence, since $\xi_{i i}(x)>0$ for all $x>0$, we have that

$$
\frac{\mathrm{d}}{\mathrm{d} x}\left(\alpha \frac{\mathrm{d} u}{\mathrm{~d} x}\right)=-\alpha \Delta_{y} u-f \quad \text { in } L^{2}\left(0, a: V^{p-3}\right)
$$

which implies that

$$
-\nabla \cdot(\alpha(x) \nabla u)=-\frac{\mathrm{d}}{\mathrm{d} x}\left(\alpha \frac{\mathrm{d} u}{\mathrm{~d} x}\right)-\alpha \Delta_{y} u=f \quad \text { in } L^{2}\left(0, a: V^{k}\right) .
$$

Finally, the boundary conditions are obviously satisfied, which concludes the proof.

Example 40 If $f \in L^{2}\left(0, a: H^{-1}(\mathcal{O})\right)$ and $u_{0}, u_{a} \in V^{1 / 2}=H_{00}^{1 / 2}(\mathcal{O})$, then $u \in \mathcal{C}([0, a]$ : $\left.H_{00}^{1 / 2}(\Omega)\right)$, since $H^{-1}(\Omega)=V^{-1}$ and $H_{00}^{1 / 2}(\Omega)=V^{1 / 2}$.

Remark 41 Once we know that the solution of (24) is the unique solution of (26) we can improve the regularity results showed in Lemma 37 and Corollary 38. Some of the regularity results for these kind of problems can be seen, for instance, in [6].

Remark 42 The above explicit formula (25) is not well-suited for computational purposes, since at $x=0$ there is a multiplication $0 \cdot \infty$. Nevertheless, we can avoid this problem by developing the right hand term, obtaining the following result

Theorem 43 Each Fourier coefficient $u_{i}, i=1, \cdots, \infty$, of the solution $u$ of the initial value problem (24) (or, equivalently, problem (26)), is given by

$$
\begin{aligned}
u_{i}(x)= & r_{i}(x)-r_{i}(a) e^{-\int_{x}^{a}\left(\alpha(t) \xi_{i i}(t)\right)^{-1} \mathrm{~d} t} \\
& +e^{-\int_{x}^{a}\left(\alpha(t) \xi_{i i}(t)\right)^{-1} \mathrm{~d} t} \int_{x}^{a} e^{\int_{s}^{a} \lambda_{i} \alpha(t) \xi_{i i}(t) \mathrm{d} t}\left(-\alpha(s) \lambda_{i} r_{i}(s)+f_{i}(s)\right) \mathrm{d} s .
\end{aligned}
$$

Proof. Let us suppose that $u_{a} \equiv 0$ (otherwise we just have to add the additional term given by the last term of the formula (25)). Integrating by parts in $(25)$, for $i=1, \cdots, \infty$, we obtain

$$
\begin{aligned}
u_{i}(x)= & r_{i}(x)-r_{i}(a) e^{-\int_{x}^{a}\left(\alpha(t) \xi_{i i}(t)\right)^{-1} \mathrm{~d} t} \\
& +e^{-\int_{x}^{a}\left(\alpha(t) \xi_{i i}(t)\right)^{-1} \mathrm{~d} t} \int_{x}^{a} e^{\int_{s}^{a}\left(\alpha(t) \xi_{i i}(t)\right)^{-1} \mathrm{~d} t} \frac{\mathrm{d} r_{i}}{\mathrm{~d} x}(s) \mathrm{d} s \\
= & r_{i}(x)-r_{i}(a) e^{-\int_{x}^{a}\left(\alpha(t) \xi_{i i}(t)\right)^{-1} \mathrm{~d} t} \\
& +e^{-\int_{x}^{a}\left(\alpha(t) \xi_{i i}(t)\right)^{-1} \mathrm{~d} t} \int_{x}^{a} e^{\int_{s}^{a} \lambda_{i} \alpha(t) \xi_{i i}(t) \mathrm{d} t}\left(-\alpha(s) \lambda_{i} r_{i}(s)+f_{i}(s)\right) \mathrm{d} s,
\end{aligned}
$$


since

$$
v=\xi_{i i} e^{\int_{s}^{a}\left(\alpha(t) \xi_{i i}(t)\right)^{-1} \mathrm{~d} t}
$$

satisfies

and therefore,

$$
\frac{\mathrm{d} v}{\mathrm{~d} x}=-v \alpha \lambda_{i} \xi
$$

$$
v(x)=e^{\int_{s}^{a} \lambda_{i} \alpha(t) \xi_{i i}(t) \mathrm{d} t}
$$

Example 44 Let $f_{i}(x) \equiv f_{i}$, constant in $\mathcal{O}$ and $\alpha \equiv 1$ in $(0, a)$. By Theorem 43 we have that the solution of problem (24) (or, equivalently, problem (26)) is

$$
\begin{aligned}
u_{i}(x)= & f_{i} \frac{\sinh \left(\sqrt{\lambda_{i}} x\right)}{\lambda_{i}}\left(e^{-\sqrt{\lambda_{i}} a}-e^{-\sqrt{\lambda_{i}} a} \frac{\sinh \left(\sqrt{\lambda_{i}} x\right)}{\sinh \left(\sqrt{\lambda_{i}} a\right)}\right. \\
& \left.+2 \arctan \left(e^{\sqrt{\lambda_{i}} a}\right)-2 \arctan \left(e^{\sqrt{\lambda_{i}} x}\right)\right) \\
& +u_{0 i}\left(e^{-\sqrt{\lambda_{i}} x}-e^{-\sqrt{\lambda_{i}} a} \frac{\sinh \left(\sqrt{\lambda_{i}} x\right)}{\sinh \left(\sqrt{\lambda_{i}} a\right)}\right)+u_{a i} \frac{\sinh \sqrt{\lambda_{i}} x}{\sinh \sqrt{\lambda_{i}} a} .
\end{aligned}
$$

This is, in fact, the solution of

$$
\left\{\begin{array}{l}
-\frac{\mathrm{d}^{2} u_{i}}{\mathrm{~d} x}(x)+\lambda_{i} u_{i}(x)=f_{i} \\
u_{i}(0)=u_{0 i}, \quad u_{i}(a)=u_{a i}
\end{array}\right.
$$

Now, let us suppose that $\Omega=(0, a) \times(0, b)$ (that is, $\mathcal{O}=(0, b)$ ). Then,

$$
\left\{\begin{array}{l}
\lambda_{n}=\left(\frac{n \pi}{b}\right)^{2}, n \geq 1 \text { and } n \in \mathbb{N}, \\
w_{n}\left(x_{2}\right)=\sqrt{\frac{2}{b}} \sin \left(\frac{n \pi}{b} x_{2}\right) .
\end{array}\right.
$$

and

$$
u\left(x, x_{2}\right)=\sqrt{\frac{2}{b}} \sum_{n=1}^{\infty} u_{n} \sin \left(\frac{n \pi}{b} x_{2}\right) .
$$

which can be approximated by

$$
u^{m}\left(x, x_{2}\right)=\sqrt{\frac{2}{b}} \sum_{n=1}^{m} u_{n} \sin \left(\frac{n \pi}{b} x_{2}\right),
$$

for $m$ large enough.

For instance, if $f \equiv 1, u_{0} \equiv 1$ and $u_{a} \equiv 1$, then

$$
f_{n}=u_{0 n}=u_{a n}= \begin{cases}\frac{2 \sqrt{2 b}}{n \pi} & \text { if } n \text { is odd, } \\ 0 & \text { if } n \text { is even. }\end{cases}
$$

Figure 2 (respectively Figure 3 ) shows the linear interpolation of $u^{20}$ (respectively $u^{50}$ ), over a grid of 16 elements for the $x$-coordinate and 26 elements for the $x_{2}$-coordinate, when $a=3$ and $b=5$. 


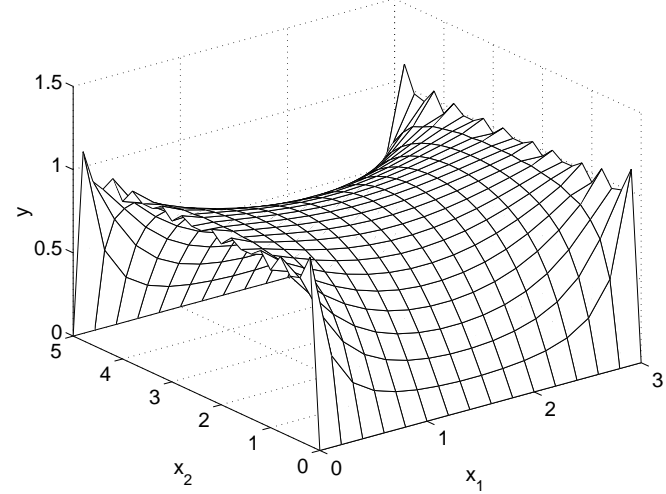

Figure 2: Graph of the function $u^{20}$ of Example 44 .

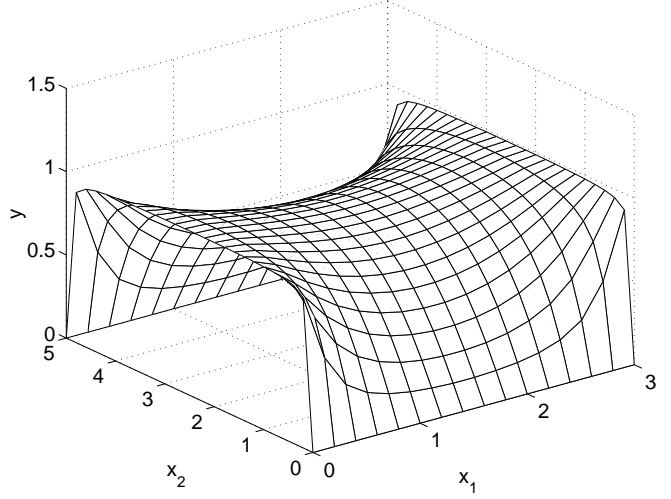

Figure 3: Graph of the function $u^{50}$ of Example 44.

\subsection{Neumann Condition on $\Gamma_{a}$}

We now change the boundary condition on $\Gamma_{a}$ to a Neumann condition. The equation for $P$ and $r$ are unchanged. Only the initial condition for $u$ on $\Gamma_{a}$ is changed.

Theorem 45 Let $j, k, q \in \mathbb{R}$ arbitrary, $u_{0} \in V^{j}, f \in L^{2}\left(0, a: V^{k}\right)$ and $u_{a} \in V^{q}$. Then, if $p=\min \{j, k+3 / 2, q+1\}$, there exists a unique solution $u \in \mathcal{C}\left([0, a]: V^{p}\right)$ of the initial value problem

$$
\left\{\begin{array}{l}
P \alpha \frac{\mathrm{d} u}{\mathrm{~d} x}=u-r, \\
u(a)=P(a) u_{a}+r(a),
\end{array}\right.
$$

where $r \in \mathcal{C}\left([0, a]: V^{\bar{p}}\right) \cap L^{2}\left(0, a: V^{\bar{p}+1 / 2} \cap H^{1}\left(0, a: V^{\bar{p}-1 / 2}\right)\right.$ is the solution of the initial value problem (19) with $\bar{p}=\min \{j, k+3 / 2\}$. Further, $u$ is also solution of the problem

$$
\begin{cases}-\nabla \cdot(\alpha(x) \nabla u)=f & \text { in } \Omega, \\ u=0 & \text { on } \Sigma, \\ u=u_{0} & \text { on } \Gamma_{0} \\ \alpha \frac{\partial u}{\partial x}=u_{a} & \text { on } \Gamma_{a}\end{cases}
$$

and each Fourier coefficient $u_{i}, i=1, \cdots, \infty$, is given by the formula of Theorem 43 , changing $u_{a}$ by $P(a) u_{a}+r(a)$.

\subsection{Transparent Boundary Conditions}

Now we show that the factorization process (2), (3) can be viewed as a Gaussian elimination process. Let us consider the subdomain $\left.\Omega_{t}^{\star}=\right] t, a[\times \mathcal{O}$. We can eliminate the unknowned function $u$ on the complementary subdomain $\left.\Omega_{t}=\right] 0, t[\times \mathcal{O}$ by writing a boundary 
value problem on $\Omega_{t}^{\star}$ with a boundary condition on $\Gamma_{t}$ thanks to the knowledge of $P(t)$ and $r(t)$. In other words this boundary condition which summarizes the behaviour of $u$ on $\Omega_{t}$ is a transparent boundary condition.

Theorem 46 Let $j, k, q \in \mathbb{R}$ arbitrary, $u_{0} \in V^{j}, f \in L^{2}\left(0, a: V^{k}\right)$ and $u_{a} \in V^{q}$. Then, if $p=\min \{j, k+3 / 2, q\}$, the unique solution $u \in \mathcal{C}\left([t, a]: V^{p}\right)$ of the initial value problem (24) (or, equivalently, the restriction of the solution of problem (26) to $\Omega_{t}^{\star}$ ), where $\left.r \in \mathcal{C}\left([0, a]: V^{\bar{p}}\right) \cap L^{2}\left(0, a: V^{\bar{p}+1 / 2}\right) \cap H^{1}\left(0, a: V^{\bar{p}-1 / 2}\right)\right)$ is the solution of the initial value problem (19) with $\bar{p}=\min \{j, k+3 / 2\}$, is also the unique solution of the elliptic boundary value problem

$$
\begin{cases}-\nabla \cdot(\alpha \nabla u)=f & \text { in } \Omega_{t}^{\star}, \\ u=0 & \text { on } \Sigma, \\ -P \alpha \frac{\partial u}{\partial x}+u=r & \text { on } \Gamma_{t}, \\ u=u_{a} & \text { on } \Gamma_{a} .\end{cases}
$$

Proof. From Corollary (38), the third equation of (29) is satisfied for $x \in[0, a]$ and, therefore, we only need to prove the uniqueness of solution of problem (29). By linearity we suppose that $f, r, u_{a}$ are equal to zero everywhere and we have to show that the only solution is $u \equiv 0$. Now, the variational formulation is

$$
\left\{\begin{array}{l}
\text { Find } u \in \mathcal{U}_{t}=\left\{\varphi \in H^{1}\left(\Omega_{t}^{\star}\right):\left.\varphi\right|_{\Sigma}=0,\left.\varphi\right|_{\Gamma_{a}}=0\right\}, \text { such that } \\
a(u, \varphi)=0 \forall \varphi \in \mathcal{U}_{t}
\end{array}\right.
$$

where

$$
a(u, \varphi)=\int_{\Omega_{t}^{\star}} \alpha \nabla u \nabla \varphi \mathrm{d} x \mathrm{~d} y+\int_{\mathcal{O}} P^{-1}(t) u(t) \varphi(t) \mathrm{d} y .
$$

Now, since $P(t)$ is a positive definite operator, it is easy to prove, by means of the Poincaré inequality, that $a(\cdot, \cdot)$ is a positive definite bilinear form on $\mathcal{U}_{t}$. This concludes the proof by using the Lax-Milgram Theorem (see Section V.3 of [3]).

Theorem 47 Let $j, k, q \in \mathbb{R}$ arbitrary, $u_{0} \in V^{j}, f \in L^{2}\left(0, a: V^{k}\right)$ and $u_{a} \in V^{q}$. Then, if $p=\min \{j, k+3 / 2, q+1\}$, the unique solution $u \in \mathcal{C}\left([0, a]: V^{p}\right)$ of the initial value problem (27) (or, equivalently, problem (28)), where $r$ is as in Theorem 46, is also the unique solution of the elliptic problem

$$
\begin{cases}-\nabla \cdot(\alpha \nabla u)=f & \text { in } \Omega_{t}^{\star} \\ u=0 & \text { on } \Sigma \\ -P \alpha \frac{\partial u}{\partial x}+u=r & \text { on } \Gamma_{t} \\ \alpha \frac{\partial u}{\partial x}=u_{a} & \text { on } \Gamma_{a}\end{cases}
$$

Proof. The proof is analogous to the proof given in Theorem 46. 
Remark 48 We consider now the problem set on the complementary domain $\Omega_{t}$.

$$
\begin{cases}-\nabla \cdot(\alpha \nabla u)=f & \text { in } \Omega_{t}, \\ u=0 & \text { on } \Sigma, \\ u=u_{0} & \text { on } \Gamma_{0}, \\ P \alpha \frac{\partial u}{\partial x}=u-r & \text { on } \Gamma_{t} .\end{cases}
$$

Let us show that this problem does not have a unique solution and therefore it is not well-posed. In fact applying Theorem 45 in $\Omega_{t}$ with an arbitrary Neumann data $h \in V^{s}$, we have that $P(t) h=P(t) \alpha \frac{\partial u}{\partial x}=u(t)-r(t)$, where $u \in \mathcal{C}\left([0, t]: V^{s+1}\right)$ is the unique solution of

$$
\begin{cases}-\nabla \cdot(\alpha(x) \nabla u)=f & \text { in } \Omega_{t}, \\ u=0 & \text { on } \Sigma, \\ u=u_{0} & \text { on } \Gamma_{0}, \\ \alpha \frac{\partial u}{\partial x}=h & \text { on } \Gamma_{t} .\end{cases}
$$

Then for any $h, u$ solution of (31) is solution of (30). By this way we recover the initial definition given in [11], [8] for operator $P$ and function $r$, before deducing its associated equations.

\section{References}

[1] Angel, E., And Bellman, R., Dynamic Programming and Partial Differential Equations. Academic Press, 1971.

[2] Balakrishnan, A. V., Functional Analysis, Springer-Verlag, New York, 1976.

[3] Brezis, H., Analyse fonctionnelle. Théorie et applications, Masson, 1983.

[4] Henry, J. And Ramos, A. M., A Direct Study of the Riccati Equation Appearing in a Factorization Method of Second Order Elliptic Boundary Value Problems by Dynamic Programming. Rapport de Recherche N. 4451 INRIA, París, 2002, ISSN: 0249-6399,(accessible at http://www.inria.fr/rrrt/rr-4451.html).

[5] Dunford, N. And Schwartz, J. T., Linear Operators. Part II: Spectral Theory, Academic Press, New York, 1963.

[6] Grisvard, P., Elliptic Problems in Nonsmooth Domains, Pitman, 1985.

[7] Hale, J. K., Ordinary Differential Equations, Wiley-Interscience, John Wiley \& Sons Inc., 1969.

[8] Henry, J. and Ramos, A. M., Factorization of Second Order Elliptic Boundary Value Problems by Dynamic Programming, Nonlinear Analysis. Theory, Methods 8 Applications, Vol. 59, 2004, pp.629-647. 
[9] Lions, J.L., Contrôle Optimal de Systèmes Gouvernés par des Équations aux Dérivées Partielles. Dunod, 1968.

[10] Lions, J. L. And Magenes, E., Problèmes aux Limites Non Homogènes et Applications, Vol. 1 and 2, Dunod, Paris, 1968.

[11] Ramos, A. M., Algunos problemas en ecuaciones en derivadas parciales relacionados con la teoría de Control. Ph.D. Thesis, Universidad Complutense de Madrid, 1996.

[12] ReID, W. T., Riccati Differential Equations, Academic Press, 1972.

[13] Temam, R., Sur l'équation de Riccati associée à des opérateurs non bornés, en dimension infinie, Journal of Functional Analysis, 7, pp. 85-115, 1971.

[14] Weyl, H., Das asymptotisch Verteilungsgesetz der Eigenwerte linearer partieller Differentialgleichungen, Math. Ann. 71, 1911, pp 441-479. 